This is an electronic reprint of the original article. This reprint may differ from the original in pagination and typographic detail.

Author(s): Ihnatsyeva, Lizaveta; Lehrbäck, Juha; Tuominen, Heli; Vähäkangas, Antti

Title: $\quad$ Fractional Hardy inequalities and visibility of the boundary

Year: $\quad 2014$

Version:

Please cite the original version:

Ihnatsyeva, L., Lehrbäck, J., Tuominen, H., \& Vähäkangas, A. (2014). Fractional Hardy inequalities and visibility of the boundary. Studia mathematica, 224(1), 47-80.

https://doi.org/10.4064/sm224-1-3

All material supplied via JYX is protected by copyright and other intellectual property rights, and duplication or sale of all or part of any of the repository collections is not permitted, except that material may be duplicated by you for your research use or educational purposes in electronic or print form. You must obtain permission for any other use. Electronic or print copies may not be offered, whether for sale or otherwise to anyone who is not an authorised user. 


\title{
FRACTIONAL HARDY INEQUALITIES AND VISIBILITY OF THE BOUNDARY
}

\author{
LIZAVETA IHNATSYEVA, JUHA LEHRBÄCK, HELI TUOMINEN, \\ AND ANTTI V. VÄHÄKANGAS
}

\begin{abstract}
We prove fractional order Hardy inequalities on open sets under a combined fatness and visibility condition on the boundary. We demonstrate by counterexamples that fatness conditions alone are not sufficient for such Hardy inequalities to hold. In addition, we give a short exposition of various fatness conditions related to our main result, and apply fractional Hardy inequalities in connection to the boundedness of extension operators for fractional Sobolev spaces.
\end{abstract}

\section{INTRODUCTION}

In this paper, we consider the following fractional $(s, p)$-Hardy inequalities:

$$
\int_{G} \frac{|u(x)|^{p}}{\operatorname{dist}(x, \partial G)^{s p}} d x \leq c \int_{G} \int_{G} \frac{|u(x)-u(y)|^{p}}{|x-y|^{n+s p}} d y d x .
$$

We say that an open set $G \subset \mathbb{R}^{n}$ admits an $(s, p)$-Hardy inequality, for $0<s<1$ and $1<p<\infty$, if there is a constant $c>0$ such that inequality (1.1) holds for every $u \in C_{0}^{\infty}(G)$. Throughout the paper, we consider only proper open subsets $G \subsetneq \mathbb{R}^{n}$, and therefore the boundary $\partial G$ is always non-empty.

One of our starting points is a result of Dyda [5] that a bounded Lipschitz domain admits an $(s, p)$-Hardy inequality if and only if $s p>1$. This can be viewed as an analogue of a result of Nečas [25], stating that if $G$ is a bounded Lipschitz domain and $1<p<\infty$, then there is $c>0$ such that the $p$-Hardy inequality

$$
\int_{G} \frac{|u(x)|^{p}}{\operatorname{dist}(x, \partial G)^{p}} d x \leq c \int_{G}|\nabla u(x)|^{p} d x
$$

holds for all $u \in C_{0}^{\infty}(G)$. For the $p$-Hardy inequality (1.2) it is well understood that the Lipschitz assumption can be weakened significantly: a canonical sufficient condition is that the complement of $G$ is $(1, p)$-uniformly fat; we refer here to the works of Lewis [22] and Wannebo [28]. On the other hand, it follows easily from the ideas in [9] and [13] that if the complement of an open set $G$ is $(s, p)$-uniformly fat, then a 'global' version of the $(s, p)$-Hardy inequality (1.1), namely the inequality

$$
\int_{G} \frac{|u(x)|^{p}}{\operatorname{dist}(x, \partial G)^{s p}} d x \leq c \int_{\mathbb{R}^{n}} \int_{\mathbb{R}^{n}} \frac{|u(x)-u(y)|^{p}}{|x-y|^{n+s p}} d y d x
$$

holds for every $u \in C_{0}^{\infty}(G)$; here we understand that functions in $C_{0}^{\infty}(G)$ are extended as 0 outside $G$; see Section 6 for more details.

The main objective of our present work is to examine how much the Lipschitz condition can be relaxed without losing the 'localization' in inequality (1.1). In

2010 Mathematics Subject Classification. Primary 46E35; Secondary 26D15.

Key words and phrases. fractional Hardy inequality, uniform fatness, visibility, Hausdorff content. 
other words, we aim to determine what kind of assumptions on $G$ allow for the restriction of the integration in the right-hand side of (1.3) to the set $G \times G$. Based on a comparison with the $p$-Hardy inequality (1.2), a natural first candidate for such a condition would be the complement of $G$ being $(s, p)$-uniformly fat. However, already the example of Dyda $[5, \S 2]$ gives an open set $G$ whose complement is $(s, p)$ uniformly fat for all $1<p<\infty$ and $0<s<1$, but where $(s, p)$-Hardy inequalities fail if $s p \leq 1$. In fact, one can simply take $G$ to be the unit ball of $\mathbb{R}^{n}$, or any other bounded Lipschitz domain. Notice that in this case the boundary of $G$ is locally $(s, p)$-uniformly fat only when $s p>1$. Hence one could ask if it is sufficient for inequality (1.1) that the boundary is (locally) $(s, p)$-uniformly fat. Again, it turns out that the answer is negative, at least in the range $0<s p \leq 1$, as we provide in Section 5 examples of open sets whose boundaries are (locally) $(s, p)$-uniformly fat, but which still fail to admit $(s, p)$-Hardy inequalities.

The obstruction in our examples is that even though the boundary is uniformly fat, most of it is not 'visible' from within the set. Hence it seems that a right way to generalize the result of Dyda [5] beyond Lipschitz domains is to apply conditions which combine both fatness and geometry of the boundary. In our main result, Theorem 4.1, we follow [17] and use a local visual boundary condition given in terms of John curves. An illustrative consequence of Theorem 4.1 is the following sufficient condition for uniform domains.

Corollary 1.4. Let $0<s<1$ and $1<p<\infty$ be such that $0<s p<n$. Assume that $G \subset \mathbb{R}^{n}$ is a (bounded) uniform domain with a (locally) ( $\left.s, p\right)$-uniformly fat boundary. Then $G$ admits an $(s, p)$-Hardy inequality.

Besides bounded Lipschitz domains (for $s p>1$ ) and domains above the graphs of Lipschitz functions (also considered by Dyda [5]), Corollary 1.4 covers, for instance, the domain $G \subset \mathbb{R}^{2}$ bounded by the usual von Koch snowflake curve of Hausdorff dimension $\lambda=\log 4 / \log 3$, or, in fact, any domain inside an analogous curve of any dimension $\lambda \in(1,2)$. It is indeed well known that these domains are uniform. Moreover, the boundary of such a domain $G$ is a $\lambda$-regular set, and therefore the boundary is locally $(s, p)$-uniformly fat for $s p>2-\lambda$ (cf. Proposition 3.15). It then follows from Corollary 1.4 that $G$ admits an $(s, p)$-Hardy inequality if (and only if) $s p>2-\lambda$. (For the converse, we refer to $[5, \S 2]$ and the estimates in Section 5.2.) Nevertheless, without going into the details in this section, we mention that Theorem 4.1 can be applied to far more general open sets than just uniform domains. Let us also remark that in the recent paper [8], related fractional Hardy inequalities are studied using different methods. One consequence of the general framework in [8] is that in Corollary 1.4 the assumption that $G$ is uniform can actually be replaced by a plumpness condition for $G$. On the other hand, our Theorem 4.1 can be applied to many non-plump cases in which the results in [8] do not apply directly, for instance to domains having outer cusps.

Fractional Hardy inequalities, and their generalizations, have been enjoying a reasonable amount of interest during the last few years. One of the reasons for this is that they can be used to deliver spectral information on the generators of the so-called censored stable processes; see e.g. [5], and the references therein, for more details. In addition to the aforementioned results and references, Loss and Sloane [23] established fractional Hardy type inequalities with sharp constants, but they used different distance functions in the left-hand side of (1.1). However, in a 
convex domain $G$ their distance functions are majorized by $\operatorname{dist}(x, \partial G)$, and thus one obtains sharp fractional inequalities (1.1) for convex domains. Dyda and Frank [7] further improved the results of Loss and Sloane into the so-called fractional HardySobolev-Mazya inequalities. See also $[2,6,10,11,26]$ for related results. For one-dimensional fractional Hardy inequalities we refer to [18, Chapter 5] and the references therein. In the present paper, we restrict ourselves completely to the case where the boundary of $G$ is 'thick', for instance in the sense of the uniform fatness condition. On the other hand, it is well-known that Hardy inequalities, in general, can be valid also when the boundary (or the complement) is 'thin' enough. For fractional Hardy inequalities, the thin case has been examined systematically in $[14,8]$; the work of Dyda [5] contains some particular instances of thin complements as well.

The outline of the rest of the paper is as follows: In Section 2 we provide definitions, notation, and other basic tools; for instance, the definition of uniform fatness, based on Riesz capacities, and the different John and uniformity conditions related to the visibility can be found here. Section 3 contains comparison results for various density conditions. In particular, we relate conditions given in terms of capacities (as in uniform fatness) to conditions for Hausdorff contents. Our main result, Theorem 4.1, is stated and proved in Section 4. The proof is based on a combination of 'pointwise Hardy' techniques developed in [17, 15, 21] and maximal function arguments, similar to those in $[9,13]$. In Section 5 we construct the counterexamples showing that $(s, p)$-uniform fatness of the boundary $\partial G$ does not suffice for $G$ to admit an $(s, p)$-Hardy inequality. We close the paper in Section 6 with some applications and additional results related to the extension of functions in the fractional Sobolev space $W^{s, p}(G)$ and to the removability of sets with respect to $(s, p)$-Hardy inequalities.

\section{Notation AND PRELIMINARIES}

2.1. Basic notation and Whitney cubes. We follow the standard convention that the letters $C$ and $c$ denote positive constants whose values are not necessarily the same at each occurrence. If there exist constants $c_{1}, c_{2}>0$ such that $c_{1} a \leq b \leq c_{2} a$, we sometimes write $a \simeq b$ and say that $a$ and $b$ are comparable.

When $E \subset \mathbb{R}^{n}$, the characteristic function of $E$ is denoted by $\chi_{E}$, the boundary of $E$ is written as $\partial E$, and $|E|$ is the $n$-dimensional Lebesgue measure of $E$. The complement of a set $G \subset \mathbb{R}^{n}$ is denoted by $G^{c}$. The integral average of a locally integrable function $f$ over a bounded set $E$ with a positive measure is written as

$$
f_{E}:=f_{E} f d x:=\frac{1}{|E|} \int_{E} f d x .
$$

The support of a function $f: \mathbb{R}^{n} \rightarrow \mathbb{C}$ is denoted by $\operatorname{spt}(f)$, and it is the closure of the set $\{x: f(x) \neq 0\}$ in $\mathbb{R}^{n}$. If $\mu$ is a Borel measure in $\mathbb{R}^{n}$, the support of $\mu$, denoted by $\operatorname{spt}(\mu)$, is the smallest closed set $E$ such that $\mu\left(\mathbb{R}^{n} \backslash E\right)=0$.

The open ball centered at $x \in \mathbb{R}^{n}$ and of radius $r>0$ is denoted by $B(x, r)$, and $Q$ always denotes a cube in $\mathbb{R}^{n}$ with sides parallel to the coordinate axes; we write $x_{Q}$ for the center of the cube $Q$ and $\ell(Q)$ for its side length. For $L>0$, we write $L Q$ for the dilated cube with side length $L \ell(Q)$ and center $x_{Q}$.

The family of closed dyadic cubes is denoted by $\mathcal{D}$, and $\mathcal{D}_{j}$ is the family of dyadic cubes of side length $2^{-j}, j \in \mathbb{Z}$. For a proper open set $G$, we fix its Whitney 
decomposition $\mathcal{W}=\mathcal{W}(G) \subset \mathcal{D}$, and write

$$
\mathcal{W}_{j}=\mathcal{W}_{j}(G):=\mathcal{W}(G) \cap \mathcal{D}_{j}
$$

for $j \in \mathbb{Z}$. Recall that $G=\cup_{Q \in \mathcal{W}(G)} Q$ and each Whitney cube $Q \in \mathcal{W}(G)$ satisfies

$$
\operatorname{diam}(Q) \leq \operatorname{dist}(Q, \partial G) \leq 4 \operatorname{diam}(Q) .
$$

For the construction and other properties of Whitney cubes we refer to [27, VI.1].

2.2. Riesz potentials and uniform fatness. The Riesz $s$-potentials, $0<s<n$, of a measurable function $f$ and a Borel measure $\mu$ on $\mathbb{R}^{n}$ are given by, respectively,

$$
\mathcal{I}_{s} f(x)=\int_{\mathbb{R}^{n}} \frac{f(y)}{|x-y|^{n-s}} d y \quad \text { and } \quad \mathcal{I}_{s} \mu(x)=\int_{\mathbb{R}^{n}} \frac{1}{|x-y|^{n-s}} d \mu(y) .
$$

If $0<s p<n$ and $1<p<\infty$, the $(s, p)$-outer capacity of a set $E$ in $\mathbb{R}^{n}$ is

$$
R_{s, p}(E)=\inf \left\{\|f\|_{p}^{p}: f \geq 0 \text { and } \mathcal{I}_{s} f \geq 1 \text { on } E\right\} .
$$

Following [22], we say that a set $E$ is locally $(s, p)$-uniformly fat for $0<s p<n$, $1<p<\infty$, if there exist positive constants $r_{0}$ and $\sigma$ such that

$$
R_{s, p}(B(x, r) \cap E) \geq \sigma r^{n-s p}
$$

for every $x \in E$ and $0<r<r_{0}$. If inequality (2.2) holds for every $x \in E$ and every $r>0$, we say that $E$ is $(s, p)$-uniformly fat.

2.3. Hausdorff measures and regular sets. The $\lambda$-Hausdorff content of a set $E \subset \mathbb{R}^{n}$ is

$$
\mathcal{H}_{\infty}^{\lambda}(E)=\inf \left\{\sum_{i=1}^{\infty} r_{i}^{\lambda}: E \subset \bigcup_{i=1}^{\infty} B\left(x_{i}, r_{i}\right), r_{i}>0\right\},
$$

where we may assume that $x_{i} \in E$, since this increases the infimum at most by a constant factor. Moreover, as is easily seen, we may allow finite coverings in the infimum above. The $\lambda$-Hausdorff measure is denoted by $\mathcal{H}^{\lambda}$, for the definition we refer to $[24, \S 4]$. Recall that the Hausdorff dimension of $E$ is the number

$$
\operatorname{dim}_{\mathcal{H}}(E)=\inf \left\{\lambda>0: \mathcal{H}_{\infty}^{\lambda}(E)=0\right\}=\inf \left\{\lambda>0: \mathcal{H}^{\lambda}(E)=0\right\} .
$$

Let $0<\lambda \leq n$. A closed set $E \subset \mathbb{R}^{n}$ is said to be an (Ahlfors) $\lambda$-regular set, or sometimes simply a $\lambda$-set, if there is a constant $C>1$ such that

$$
C^{-1} r^{\lambda} \leq \mathcal{H}^{\lambda}(B(x, r) \cap E) \leq C r^{\lambda}
$$

for every $x \in E$ and all $0<r<\operatorname{diam}(E)$.

2.4. John domains and visual boundary. We say that a domain (an open and connected set) $G \subset \mathbb{R}^{n}$ is a $c$-John domain, $c \geq 1$, with center point $x_{0}$, if for every $x \in G$ there exists a curve (called a John curve) $\gamma:[0, \ell] \rightarrow G$, parameterized by arc length, such that $\gamma(0)=x, \gamma(\ell)=x_{0}$, and

$$
\operatorname{dist}(\gamma(t), \partial G) \geq \frac{1}{c} t
$$

for each $t \in[0, \ell]$. If $G$ is a $c$-John domain with center point $x_{0}$, then

$$
G \subset B\left(x_{0}, c \operatorname{dist}\left(x_{0}, \partial G\right)\right) ;
$$

in particular, $G$ is bounded. Also, if $G$ is a $c$-John domain, then for each $w \in \partial G$ there is a curve $\gamma:[0, \ell] \rightarrow G \cup\{w\}$ joining $w$ to $x_{0}$ and satisfying (2.4). We say in this case, too, that $\gamma$ joins $w$ to $x_{0}$ in $G$. 
When $G \subset \mathbb{R}^{n}$ is an open set, $x \in G$, and $c \geq 1$ is a constant, we define a subdomain $G_{x, c}$ by

$$
G_{x, c}=\bigcup\{U \subset G: U \text { is a } c \text {-John domain with center point } x\} .
$$

Then clearly $\emptyset \neq G_{x, c} \subset G$ and $G_{x, c}$ is also a $c$-John domain with center point $x$. Following [17], we say that the set

$$
\partial_{x, c}^{\mathrm{vis}} G:=\partial G \cap \partial G_{x, c}
$$

is the c-visual boundary of $G$ near $x$.

A domain $G \subset \mathbb{R}^{n}$ is a uniform domain if there is a constant $C \geq 1$ such that each pair of points $x, y \in G$ can be joined by a curve $\gamma:[0, \ell] \rightarrow G$, parameterized by arc length, so that $\ell \leq C|x-y|$ and $\operatorname{dist}(z, \partial G) \geq \frac{1}{C} \min \{|z-x|,|z-y|\}$ for each $z \in \gamma$. Every bounded uniform domain is also a $c$-John domain for some $c \geq 1$.

\section{Comparison Results}

In this expository section, we study the connections between uniform fatness and thickness conditions formulated in terms of Hausdorff contents; thereby we clarify the relations of the conditions which commonly appear in connection to our main theorem.

More precisely, in our main result, Theorem 4.1, we assume that the visual boundary is uniformly large near each point $x \in G$, in the sense of the $\lambda$-Hausdorff content for an appropriate exponent $\lambda>0$, as follows:

$$
\mathcal{H}_{\infty}^{\lambda}\left(\partial_{x, c}^{\text {vis }} G\right) \geq C_{0} \operatorname{dist}(x, \partial G)^{\lambda},
$$

where $C_{0}>0$ and $c \geq 1$ are independent of $x$. A closely related condition is an inner boundary density condition with an exponent $\lambda$, which is satisfied by an open set $G \subset \mathbb{R}^{n}$ if there exists a constant $C>0$ such that

$$
\mathcal{H}_{\infty}^{\lambda}(\partial G \cap B(x, 2 \operatorname{dist}(x, \partial G))) \geq C \operatorname{dist}(x, \partial G)^{\lambda}
$$

for all $x \in G$ (cf. [19]).

Our main assumption (3.1) is stronger than the inner boundary density condition (3.2) with the same exponent $\lambda$. Indeed, one may apply relation (2.5) and the observation that an open set $G \subset \mathbb{R}^{n}$ satisfies condition (3.1) if and only if for each $x \in G$ there exists some $c$-John domain $U_{x} \subset G$, with center point $x$, such that

$$
\mathcal{H}_{\infty}^{\lambda}\left(\partial U_{x} \cap \partial G\right) \geq C_{0} \operatorname{dist}(x, \partial G)^{\lambda} .
$$

On the other hand, there is a wide class of domains for which a converse is true as well. For instance, a uniform domain $G$ satisfies condition (3.1) if and only if $G$ satisfies condition (3.2). In fact, for uniform domains the visual boundary near $x$ coincides with the boundary (near $x$ ), as the following adaptation of $[17$, Proposition 4.3] shows.

Proposition 3.3. Assume that $G \subset \mathbb{R}^{n}$ is a uniform domain. Then there exists a constant $c \geq 1$, depending only on $n$ and the constant in the uniformity condition for $G$, such that for all $x \in G$

$$
\partial G \cap B(x, 2 \operatorname{dist}(x, \partial G)) \subset \partial_{x, c}^{\mathrm{vis}} G .
$$


The inner boundary density condition (3.2) is satisfied with an exponent $\lambda>$ $n-s p$, e.g. in the case of a (bounded) open set with a (locally) $(s, p)$-uniformly fat boundary, where $1<p<\infty$ and $0<s p<n$. This fact is a consequence of Proposition 3.7 below. Nevertheless, we remark that the fatness of the boundary is not necessary for either of the conditions (3.1) and (3.2) to hold, as domains with outward cusps show.

More generally, the following Theorem 3.4 gives a precise connection between the inner boundary density condition and the uniform fatness of the complement of an open set; as indicated, the case $0<s p \leq 1$ is of particular interest. The theorem mostly restates, and also slightly extends, some of the results obtained in [19].

Theorem 3.4. Let $1<p<\infty$ and $0<s p<n$, and consider the following conditions for an open set $G \subset \mathbb{R}^{n}$ :

(1) the complement $G^{c}$ is $(s, p)$-uniformly fat;

(2) there exists $n-s p<\lambda \leq n$ and $C>0$ such that, for all $r>0$ and for every $x \in G^{c}, \mathcal{H}_{\infty}^{\lambda}\left(B(x, r) \cap G^{c}\right) \geq C r^{\lambda}$;

(3) $G$ satisfies (3.2) with an exponent $n-s p<\lambda \leq n$.

Then conditions (1) and (2) are (quantitatively) equivalent, and condition (3) implies (1) and (2). Moreover, for $s p>1$, all of the conditions (1)-(3) are equivalent; here the assumption $s p>1$ cannot be relaxed in general.

Proof. The equivalence $(1) \Leftrightarrow(2)$ is a consequence of Propositions 3.7 and 3.12 proved in Sections 3.1 and 3.2 below. We remark that these propositions are most likely known to the experts, and they are based on ideas in $[1, \S 5]$, but we include some details for the sake of exposition.

The implication $(3) \Rightarrow(2)$ is proved in [19, pp. 2197-2198]. Furthermore, if $s p>1$, then the converse implication follows by a careful inspection of the proofs in [19]; note that in [19] the claims are formulated for domains, but they actually hold for all open sets. The ball $G=B(0,1)$ serves as a counterexample, showing that the assumption $s p>1$ is necessary for the implication $(2) \Rightarrow(3)$ to hold in general (recall that $\partial G$ has Hausdorff dimension $n-1$, hence $\mathcal{H}_{\infty}^{\lambda}(\partial G)=0$ if $\left.n-1<\lambda \leq n\right)$.

3.1. Uniform fatness $\Rightarrow$ thickness. (By 'thickness' we refer to density in terms of Hausdorff contents, see condition (2) in Theorem 3.4.)

We begin with an easy bound for Riesz capacities in terms of Hausdorff contents:

Lemma 3.5. If $1<p<\infty$ and $0<s p<n$, then there is a constant $C>0$ such that $R_{s, p}(E) \leq C \mathcal{H}_{\infty}^{n-s p}(E)$ for all sets $E \subset \mathbb{R}^{n}$.

Proof. Let $E \subset \bigcup_{i} B_{i}$, where $B_{i}=B\left(x_{i}, r_{i}\right)$ with $x_{i} \in \mathbb{R}^{n}, r_{i}>0$. By the monotonicity and the subadditivity of the Riesz capacity, we have

$$
R_{s, p}(E) \leq R_{s, p}\left(\bigcup_{i} B_{i}\right) \leq \sum_{i} R_{s, p}\left(B_{i}\right)
$$

Since $R_{s, p}\left(B_{i}\right) \leq C r_{i}^{n-s p}$ for all balls, [1, Proposition 5.1.2], the claim follows by taking the infimum over all such covers of $E$.

The following deep theorem from [22] states that local uniform fatness of closed sets is a self-improving property. 
Theorem 3.6. Let $0<s p<n$ and $1<p<\infty$. Assume that $E \subset \mathbb{R}^{n}$ is closed and locally $(s, p)$-uniformly fat (with constant $\sigma>0$ ). Then there are constants $\varepsilon, \sigma_{1}>0$, depending only on $s, p, n, \sigma$, such that $R_{\beta, q}(B(x, r) \cap E) \geq \sigma_{1} r^{n-\beta q}$ whenever $x \in E$, $0<r<r_{0}$, and $s p-\varepsilon<\beta q<s p$.

In other words, a closed and locally $(s, p)$-uniformly fat set $E$ is actually $(\beta, q)$ locally uniformly fat also for $s p-\varepsilon<\beta q \leq s p$. Since the constants $\varepsilon$ and $\sigma_{1}$ in the formulation of Theorem 3.6 are independent of the parameter $r_{0}$, we see that uniform fatness of closed sets is self-improving as well. With the help of the selfimprovement we obtain the following proposition, which yields in particular the implication $(1) \Rightarrow(2)$ of Theorem 3.4.

Proposition 3.7. Let $1<p<\infty$ and $0<s p<n$. Assume that $E \subset \mathbb{R}^{n}$ is closed and $(s, p)$-uniformly fat. Then there exist $n-s p<\lambda \leq n$ and $C>0$ such that

$$
\mathcal{H}_{\infty}^{\lambda}(B(x, r) \cap E) \geq C r^{\lambda}
$$

for every $x \in E$ and for all $r>0$.

Proof. By the self-improvement, there is $1<q<p$ such that $E$ is $(s, q)$-uniformly fat. It follows from the definition of $(s, q)$-uniform fatness and Lemma 3.5 that

$$
r^{n-s q} \leq \sigma_{1}^{-1} R_{s, q}(B(x, r) \cap E) \leq C \mathcal{H}_{\infty}^{n-s q}(B(x, r) \cap E)
$$

for every $x \in E$ and all $r>0$. Hence the claim follows with $\lambda=n-s q>n-s p$.

Note that if $E$ is locally $(s, p)$-uniformly fat, then the claim of Proposition 3.7 holds for all $0<r<r_{0}$.

3.2. Thickness $\Rightarrow$ uniform fatness. Suppose $E \subset \mathbb{R}^{n}, 1<p<\infty$, and $0<$ $s p<n$. As in [24], $\mathcal{M}(E)$ denotes the set of Radon measures with compact support satisfying $\operatorname{spt}(\mu) \subset E$ and $0<\mu\left(\mathbb{R}^{n}\right)<\infty$. Below we use the following variant of Frostman's lemma from [24, Theorem 8.8]; see also [3].

Lemma 3.8. Let $E$ be a Borel set in $\mathbb{R}^{n}$ and let $\lambda>0$. Then $\mathcal{H}^{\lambda}(E)>0$ if and only if there exists $\mu \in \mathcal{M}(E)$ such that $\mu(B(x, r)) \leq r^{\lambda}$ for $x \in \mathbb{R}^{n}$ and $r>0$. Moreover, we can find $\mu$ so that $\mu(E) \geq C_{n} \mathcal{H}_{\infty}^{\lambda}(E)$ where $C_{n}>0$ depends only on $n$.

The Wolff potential of a measure $\mu \in \mathcal{M}\left(\mathbb{R}^{n}\right)$ at $y \in \mathbb{R}^{n}$ is

$$
\dot{W}_{s, p}^{\mu}(y)=\int_{0}^{\infty}\left(\frac{\mu(B(y, t))}{t^{n-s p}}\right)^{p^{\prime}-1} \frac{d t}{t}, \quad p^{\prime}=p /(p-1) .
$$

By Wolff's inequality, there is $c>0$ such that

$$
\sup \mu(E) \leq c R_{s, p}(E)
$$

where the supremum is taken over $\mu \in \mathcal{M}(E)$ satisfying $\dot{W}_{s, p}^{\mu}(y) \leq 1$ for all $y \in \mathbb{R}^{n}$.

Remark 3.10. Although inequality (3.9) is well known and widely used, it is worthwhile to sketch a proof. Here it is convenient to use a dual definition for the Riesz capacity of a compact set $K \subset E$, statement (A) in [22, p. 177]:

$$
R_{s, p}(K)=\sup \left\{v(K)^{p}: \operatorname{spt}(v) \subset K \text { and }\left\|\mathcal{I}_{s} v\right\|_{p^{\prime}} \leq 1\right\}
$$


Let $\mu \in \mathcal{M}(E)$ be a measure such that $\dot{W}_{s, p}^{\mu}(y) \leq 1$ for every $y \in \mathbb{R}^{n}$. Write $K=\operatorname{spt}(\mu)$. By Wolff's inequality, [1, Theorem 4.5.4], there is $A>0$, depending on $s, p$, and $n$, such that

$$
\int_{\mathbb{R}^{n}}\left|\mathcal{I}_{s} \mu\right|^{p^{\prime}} d x=\int_{\mathbb{R}^{n}}\left(\mathcal{I}_{s} \mu\right)^{p^{\prime}} d x \leq A \int_{\mathbb{R}^{n}} \dot{W}_{s, p}^{\mu} d \mu \leq A \mu(K) .
$$

Define $v:=A^{-1 / p^{\prime}} \mu(K)^{-1 / p^{\prime}} \mu \in \mathcal{M}(K)$. By inequality (3.11), the measure $v$ is admissible for the dual definition of capacity. Since $K \subset E$, we have that

$$
R_{s, p}(E) \geq R_{s, p}(K) \geq v(K)^{p}=A^{-p / p^{\prime}} \mu(K)^{-p / p^{\prime}} \mu(K)^{p}=A^{-p / p^{\prime}} \mu(E) .
$$

Taking the supremum over measures $\mu$ yields inequality (3.9) with the implied constant $A^{p / p^{\prime}}$.

The following proposition shows that thickness implies uniform fatness; in particular, $(2) \Rightarrow(1)$ holds in Theorem 3.4. For a closely related comparison theorem, see [1, Corollary 5.1.14].

Proposition 3.12. Let $s, p, \lambda$ be such that $1<p<\infty, 0<s p<n$, and $n-s p<$ $\lambda \leq n$. Let $E \subset \mathbb{R}^{n}$ be a Borel set such that for a constant $\sigma>0$,

$$
\mathcal{H}_{\infty}^{\lambda}(B(x, r) \cap E) \geq \sigma r^{\lambda}
$$

for all $x \in E$ and $r>0$. Then $E$ is $(s, p)$-uniformly fat.

Proof. Let $x \in E, r>0$, and let

$$
E_{x, r}=B(x, r) \cap E .
$$

By condition (3.13), we find that $\mathcal{H}^{\lambda}\left(E_{x, r}\right)>0$. Frostman's Lemma 3.8 yields a measure $\widetilde{\mu} \in \mathcal{M}\left(E_{x, r}\right)$ satisfying $\widetilde{\mu}(B(y, t)) \leq t^{\lambda}$ for each $y \in \mathbb{R}^{n}$ and $t>0$. Moreover,

$$
\widetilde{\mu}\left(E_{x, r}\right) \geq C_{n} \mathcal{H}_{\infty}^{\lambda}\left(E_{x, r}\right),
$$

where $C_{n}>0$ depends only on $n$.

Now, for a fixed $y \in \mathbb{R}^{n}$, we write

$$
\dot{W}_{s, p}^{\widetilde{\mu}}(y)=\left\{\int_{0}^{r}+\int_{r}^{\infty}\right\}\left(\frac{\widetilde{\mu}(B(y, t))}{t^{n-s p}}\right)^{p^{\prime}-1} \frac{d t}{t}=: A+B .
$$

We first estimate term $A$ using the properties of the Frostman measure $\widetilde{\mu}$ :

$$
\begin{aligned}
A & =\int_{0}^{r}\left(\frac{\widetilde{\mu}(B(y, t))}{t^{n-s p}}\right)^{p^{\prime}-1} \frac{d t}{t} \\
& \leq \int_{0}^{r} t^{(\lambda-n+s p)\left(p^{\prime}-1\right)-1} d t=\frac{r^{(\lambda-n+s p)\left(p^{\prime}-1\right)}}{(\lambda-n+s p)\left(p^{\prime}-1\right)} .
\end{aligned}
$$

Next term $B$ is considered. We begin with a preliminary observation: for $y \in \mathbb{R}^{n}$ and $t>0$,

$$
\widetilde{\mu}(B(y, t))=\widetilde{\mu}(B(y, t) \cap B(x, r)) \leq \widetilde{\mu}(B(x, r)) \leq r^{\lambda} .
$$

This follows from the fact that $\widetilde{\mu}$ is supported inside $E_{x, r} \subset B(x, r)$. Thus we obtain

$$
B \leq r^{\lambda\left(p^{\prime}-1\right)} \int_{r}^{\infty} t^{(s p-n)\left(p^{\prime}-1\right)-1} d t=\frac{r^{\lambda\left(p^{\prime}-1\right)+(s p-n)\left(p^{\prime}-1\right)}}{(n-s p)\left(p^{\prime}-1\right)}=\frac{r^{(\lambda-n+s p)\left(p^{\prime}-1\right)}}{(n-s p)\left(p^{\prime}-1\right)} .
$$

The above estimates show that $\dot{W}_{s, p}^{\widetilde{\mu}}(y)=A+B \leq \kappa r^{(\lambda-n+s p)\left(p^{\prime}-1\right)}$, where the positive constant $\kappa$ depends on $n, s, p$, and $\lambda$. 
Let us define $\mu:=\kappa^{-1 /\left(p^{\prime}-1\right)} r^{n-s p-\lambda} \widetilde{\mu}$. Then $\mu \in \mathcal{M}\left(E_{x, r}\right)$ and, for every $y \in \mathbb{R}^{n}$,

$$
\dot{W}_{s, p}^{\mu}(y)=\kappa^{-1} r^{(n-s p-\lambda)\left(p^{\prime}-1\right)} \dot{W}_{s, p}^{\widetilde{\mu}}(y) \leq 1 .
$$

Hence, by assumption (3.13) and inequalities (3.14) and (3.9),

$$
\sigma r^{\lambda} \leq \mathcal{H}_{\infty}^{\lambda}\left(E_{x, r}\right) \leq C_{n}^{-1} \widetilde{\mu}\left(E_{x, r}\right) \leq c C_{n}^{-1} \kappa^{1 /\left(p^{\prime}-1\right)} r^{\lambda-n+s p} R_{s, p}\left(E_{x, r}\right) .
$$

After a simplification of the exponents we find that

$$
C_{\sigma, \lambda, n, s, p} r^{n-s p} \leq R_{s, p}\left(E_{x, r}\right)=R_{s, p}(B(x, r) \cap E) .
$$

This concludes the proof.

Note again that if the thickness condition (3.13) holds for all $x \in E$ and every $0<r<r_{0}$, then $E$ is locally $(s, p)$-uniformly fat.

3.3. $\lambda$-regular sets and uniform fatness. The following result relating $\lambda$-regular sets and uniform fatness is useful from the viewpoint of applications.

Proposition 3.15. Let $s, p, \lambda$ be such that $1<p<\infty, 0<s p<n$, and $n-s p<\lambda \leq$ $n$. If $E \subset \mathbb{R}^{n}$ is an unbounded $\lambda$-regular set, then $E$ is $(s, p)$-uniformly fat. On the other hand, if $E \subset \mathbb{R}^{n}$ is a bounded $\lambda$-regular set, then $E$ is locally $(s, p)$-uniformly fat.

Proof. Let us consider the unbounded case; the bounded case is similar. Let $x \in E$, $r>0$, and let $E_{x, r}=\bar{B}(x, r / 2) \cap E$. Since $\lambda$-regular sets are closed (by definition), the set $E_{x, r}$ is compact, and we may consider the measure

$$
\widetilde{\mu}=\left.\mathcal{H}^{\lambda}\right|_{E_{x, r}} \in \mathcal{M}\left(E_{x, r}\right) .
$$

For the last relation, we refer to [24, p. 57]. By the regularity condition (2.3), for each $y \in \mathbb{R}^{n}$ and for all $0<t \leq r$ we have

$$
\widetilde{\mu}(B(y, t)) \leq C t^{\lambda}
$$

and, moreover, $\widetilde{\mu}\left(\mathbb{R}^{n}\right)=\widetilde{\mu}\left(E_{x, r}\right) \simeq r^{\lambda}$. Hence, proceeding as in the proof of Proposition 3.12 , we obtain

$$
r^{n-s p} \leq c R_{s, p}\left(E_{x, r}\right) \leq c R_{s, p}(B(x, r) \cap E) .
$$

This concludes the proof, as $x \in E$ and $r>0$ are arbitrary.

\section{Main Theorem}

The following sufficient condition for fractional Hardy inequalities is the main result of this paper.

Theorem 4.1. Let $0<s<1$ and $1<p<\infty$ satisfy $0<s p<n$, and let $G \subset \mathbb{R}^{n}$ be an open set. Assume that there exists $n-s p<\lambda \leq n$ and $C_{0}>0, c \geq 1$ such that

$$
\mathcal{H}_{\infty}^{\lambda}\left(\partial_{x, c}^{\text {vis }} G\right) \geq C_{0} \operatorname{dist}(x, \partial G)^{\lambda}
$$

for all $x \in G$. Then $G$ admits an $(s, p)$-Hardy inequality.

The proof of Theorem 4.1 is based upon a general scheme, built in [9, 13], in combination with visual boundary and pointwise Hardy techniques, developed in [17, 15, 21]. For the application of the latter in the present setting, we need the following fractional Poincaré-type inequalities: 
Lemma 4.3. Let $Q$ be a cube in $\mathbb{R}^{n}, n \geq 2$, and let $p, q \in[1, \infty), \beta \in(0,1)$, and $1 / q-1 / p<\beta / n$. Then the following fractional $(p, q, \beta)$-Poincaré inequality holds for every $u \in L^{q}(Q)$ :

$$
\int_{Q}\left|u(x)-u_{Q}\right|^{p} d x \leq c|Q|^{1+p \beta / n-p / q}\left(\int_{Q} \int_{Q} \frac{|u(x)-u(y)|^{q}}{|x-y|^{n+\beta q}} d y d x\right)^{p / q}
$$

Here the constant $c>0$ is independent of $Q$ and $u$.

Regarding the proof of Lemma 4.3, in the Sobolev-Poincaré case $p>q$ we apply scaling and translation invariance, and thereby reduce to the unit cube $[-1 / 2,1 / 2]^{n}$. For the unit cube, the claim follows from [12, Remark 4.14]. If $p \leq q$, we apply the previous case and Hölder's inequality.

The following key estimate yields a connection between the size of the visual boundary and the double integrals appearing in the right-hand sides of the fractional Hardy inequalities.

Lemma 4.4. Let $G \subset \mathbb{R}^{n}$ be an open set. Assume that $0<\beta<1,1 \leq q<\infty$, and $n-\beta q<\lambda \leq n$. Fix $c \geq 1$ and let $Q \in \mathcal{W}(G)$. Then there exist constants $L=L(c, n) \geq 1$ and $C=C(n, c, \lambda, q, \beta)>0$ (both independent of $Q$ ), such that

$$
\mathcal{H}_{\infty}^{\lambda}\left(\partial_{x_{Q}, c}^{\mathrm{vis}} G\right)\left|u_{Q}\right|^{q} \leq C \ell(Q)^{\lambda-n+\beta q} \int_{L Q \cap G} \int_{L Q \cap G} \frac{|u(x)-u(y)|^{q}}{|x-y|^{n+\beta q}} d y d x
$$

for every $u \in C_{0}^{\infty}(G)$.

Proof. Throughout the proof, $C$ denotes a positive constant, whose value may change from one occurrence to another, and which depends at most on $n, c, \lambda, q$, and $\beta$.

Let $u \in C_{0}^{\infty}(G)$. If $\left|u_{Q}\right|=0$, the claim is trivial, and thus we may assume $\left|u_{Q}\right| \neq 0$. Moreover, by homogeneity, we may assume $\left|u_{Q}\right|=1$ (otherwise just consider the function $\left.v=u /\left|u_{Q}\right|\right)$.

Let $w \in \partial_{x_{Q}, c}^{\text {vis }} G$, and let $\gamma$ be a $c$-John curve connecting $w$ to the center point $x_{Q}$ in $G_{x_{Q}, c} \subset G$. We construct a chain of $\mathcal{W}(G)$-cubes $C(Q, w)=\left(Q_{0}, Q_{1}, \ldots\right)$ joining $Q_{0}=Q$ to $\omega$ as follows. First, let us set $t_{0}=\ell$, where $\gamma(\ell)=x_{Q}$. Then, in the inductive stage, if cubes $Q_{0}, \ldots, Q_{i}$ and numbers $t_{0}, \ldots, t_{i}$ are chosen, we let $0<t_{i+1}<t_{i}$ be the smallest number for which $\gamma\left(t_{i+1}\right)$ belongs to a cube $Q^{\prime} \in \mathcal{W}(G)$ which intersects $Q_{i}$. We set $Q_{i+1}=Q^{\prime}$. Then, by the construction, $\gamma\left(t_{i}\right) \in Q_{i}$ and $\gamma(t)$, for $0<t<t_{i+1}$, does not belong to any cube $Q^{\prime \prime} \in \mathcal{W}(G)$ intersecting $Q_{i}$. In particular,

$$
\left|\gamma\left(t_{i}\right)-\gamma\left(t_{i+1}\right)\right| \geq \ell\left(Q_{i}\right) / 5
$$

since, by $(2.1), \ell\left(Q_{i}\right) / 5 \leq \ell\left(Q^{\prime \prime}\right)$ for all such cubes $Q^{\prime \prime}$. By the arc-length parametrization of $\gamma$ we thus obtain

$$
t_{i}-t_{i+1} \geq\left|\gamma\left(t_{i}\right)-\gamma\left(t_{i+1}\right)\right| \geq \ell\left(Q_{i}\right) / 5 \quad \text { for all } i \geq 0 .
$$

Next we show that

$$
\lim _{i \rightarrow \infty} u_{Q_{i}}=u(w)=0 .
$$

To this end, for $i \geq 0$ we first estimate

$$
\begin{aligned}
\operatorname{diam}\left(Q_{i}\right) & \leq \operatorname{dist}\left(Q_{i}, \partial G\right) \leq\left|\gamma\left(t_{i}\right)-w\right|=\left|\gamma\left(t_{i}\right)-\gamma(0)\right| \\
& \leq t_{i} \leq c \operatorname{dist}\left(\gamma\left(t_{i}\right), \partial G\right) \leq 5 c \operatorname{diam}\left(Q_{i}\right) .
\end{aligned}
$$


By inequalities (4.6) and (4.8), we find that

$$
t_{i}-t_{i+1} \geq \ell\left(Q_{i}\right) / 5 \geq t_{i} /(25 c \sqrt{n}),
$$

where $C(n, c)=(25 c \sqrt{n})^{-1} \in(0,1)$, and thus

$$
t_{i} \leq(1-C(n, c))^{i} t_{0} \stackrel{i \rightarrow \infty}{\longrightarrow} 0 .
$$

To conclude the proof of (4.7), it suffices to observe that $Q_{i} \subset G \cap B\left(w, 3 t_{i}\right)$; indeed, by the arc-length parametrization of $\gamma$ and estimate (4.8) we obtain, for every $y \in Q_{i}$, that

$$
|y-w| \leq\left|y-\gamma\left(t_{i}\right)\right|+\left|\gamma\left(t_{i}\right)-\gamma(0)\right| \leq \operatorname{diam}\left(Q_{i}\right)+t_{i} \leq 2 t_{i}<3 t_{i} .
$$

Observe also that (4.9) implies

$$
\sum_{i=0}^{\infty} \ell\left(Q_{i}\right)^{\delta} \leq \sum_{i=0}^{\infty} t_{i}^{\delta} \leq t_{0}^{\delta} \sum_{i=0}^{\infty}(1-C(n, c))^{i \delta} \leq C(n, c, \delta) \ell\left(Q_{0}\right)^{\delta}
$$

for any $\delta>0$.

Let $Q_{i}, Q_{i+1}$ be two consecutive cubes in the chain $C(Q, w)$. By construction, these are two intersecting Whitney cubes. Let us first assume that $\ell\left(Q_{i}\right) \geq \ell\left(Q_{i+1}\right)$. Then both cubes are contained in $3 Q_{i}$ and their measures are comparable with a constant depending on $n$ only. Note also that by $(2.1)$ we have $\operatorname{int}\left(3 Q_{i}\right) \subset G$. By the fractional $(1, q, \beta)$-Poincaré inequality for cubes, Lemma 4.3,

$$
\begin{aligned}
\left|u_{Q_{i}}-u_{Q_{i+1}}\right| & \leq\left|u_{Q_{i}}-u_{3 Q_{i}}\right|+\left|u_{3 Q_{i}}-u_{Q_{i+1}}\right| \\
& \leq C\left|3 Q_{i}\right|^{-1} \int_{3 Q_{i}}\left|u(x)-u_{3 Q_{i}}\right| d x \\
& \leq C\left|3 Q_{i}\right|^{\beta / n-1 / q}\left(\int_{3 Q_{i}} \int_{3 Q_{i}} \frac{|u(x)-u(y)|^{q}}{|x-y|^{n+\beta q}} d y d x\right)^{1 / q} .
\end{aligned}
$$

In case of $\ell\left(Q_{i}\right)<\ell\left(Q_{i+1}\right)$, we have the same estimate but with $3 Q_{i+1}$ on the last line. Since $u(w)=0$ and $\left|u_{Q}\right|=1$, it follows from (4.7) that

$$
\begin{aligned}
1 & =\left|u(w)-u_{Q}\right| \leq \sum_{i=0}^{\infty}\left|u_{Q_{i}}-u_{Q_{i+1}}\right| \\
& \leq C \sum_{i=0}^{\infty} \ell\left(Q_{i}\right)^{\beta-n / q}\left(\int_{3 Q_{i}} \int_{3 Q_{i}} \frac{|u(x)-u(y)|^{q}}{|x-y|^{n+\beta q}} d y d x\right)^{1 / q} .
\end{aligned}
$$

Comparison of the sums in (4.10) and (4.12) leads to the observation that, for each $\delta>0$, there exists an index $i_{w} \geq 0$ such that

$$
\left(\frac{\ell\left(Q_{i_{w}}\right)}{\ell(Q)}\right)^{\delta} \leq C \ell\left(Q_{i_{w}}\right)^{\beta-n / q}\left(\int_{3 Q_{i_{w}}} \int_{3 Q_{i_{w}}} \frac{|u(x)-u(y)|^{q}}{|x-y|^{n+\beta q}} d y d x\right)^{1 / q} .
$$

We now choose $\delta=(\lambda-n+\beta q) / q>0$, and so, writing $Q_{i_{w}}=Q_{w}$, we have found a cube $Q_{w} \in C(Q, w)$ satisfying

$$
\ell\left(Q_{w}\right)^{\lambda} \leq C \ell(Q)^{\lambda-n+\beta q} \int_{3 Q_{w}} \int_{3 Q_{w}} \frac{|u(x)-u(y)|^{q}}{|x-y|^{n+\beta q}} d y d x .
$$


Next we claim that $3 Q_{w} \subset L Q$, where $L=L(c, n)=30 c \sqrt{n} \geq 1$. Indeed, if $y \in 3 Q_{w}$, then inequalities (4.8) and the arc-length parametrization of $\gamma$ imply that

$$
\begin{aligned}
\left|y-x_{Q}\right| & \leq\left|y-\gamma\left(t_{i_{w}}\right)\right|+\left|\gamma\left(t_{i_{w}}\right)-\gamma\left(t_{0}\right)\right| \\
& \leq \operatorname{diam}\left(3 Q_{w}\right)+t_{0}-t_{i_{w}} \leq 3 t_{0} \leq 15 c \operatorname{diam}(Q)=30 c \sqrt{n} \ell(Q) / 2
\end{aligned}
$$

and hence $3 Q_{w} \subset L Q$. Analogous estimates also show that

$$
w \in B_{w}:=B\left(x_{Q_{w}}, \rho \ell\left(Q_{w}\right)\right) \text { and } 3 Q_{\omega} \subset B_{\omega},
$$

where $\rho=\rho(c, n)=11 c \sqrt{n}>0$.

We can now finish the proof. Since a cube as in (4.13) exists for each $w \in \partial_{x_{Q}, c}^{\text {vis }} G$,

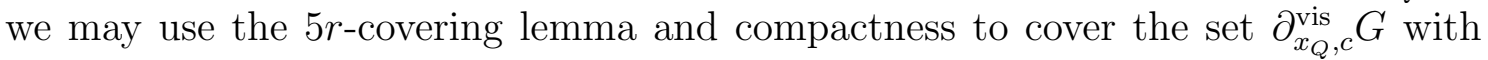
balls $5 B_{w_{j}}$, centered in $w_{i} \in \partial_{x_{Q}, c}^{\text {vis }} G, j=1, \ldots, N$, in such a way that the balls $B_{j}=B_{w_{j}}$ are pairwise disjoint and $\partial_{x_{Q}, c}^{\text {vis }} G \subset \bigcup_{j=1}^{N} 5 B_{j}$.

Let us write $Q^{j}=Q_{w_{j}}$. Now estimate (4.13) and the pairwise disjointness (since $\left.3 Q^{j} \subset B_{j}\right)$ of the cubes $3 Q^{j}$, satisfying $\operatorname{int}\left(3 Q^{j}\right) \subset L Q \cap G$, lead to

$$
\begin{aligned}
\mathcal{H}_{\infty}^{\lambda}\left(\partial_{x_{Q}, c}^{\text {vis }} G\right) & \leq \sum_{j=1}^{N}\left(5 \rho \ell\left(Q^{j}\right)\right)^{\lambda} \leq C \sum_{j=1}^{N} \ell\left(Q^{j}\right)^{\lambda} \\
& \leq C \sum_{j=1}^{N} \ell(Q)^{\lambda-n+\beta q} \int_{3 Q^{j}} \int_{3 Q^{j}} \frac{|u(x)-u(y)|^{q}}{|x-y|^{n+\beta q}} d y d x \\
& \leq C \sum_{j=1}^{N} \ell(Q)^{\lambda-n+\beta q} \int_{3 Q^{j}} \int_{L Q \cap G} \frac{|u(x)-u(y)|^{q}}{|x-y|^{n+\beta q}} d y d x \\
& \leq C \ell(Q)^{\lambda-n+\beta q} \int_{L Q \cap G} \int_{L Q \cap G} \frac{|u(x)-u(y)|^{q}}{|x-y|^{n+\beta q}} d y d x .
\end{aligned}
$$

As we assumed $\left|u_{Q}\right|=1$, the claim follows from (4.15).

The next lemma, essentially [13, Lemma 3.4], is also needed in the proof of Theorem 4.1. For the sake of convenience, we include also a proof. Notice that inequality (4.17) is not a direct consequence of Hölder's inequality, since the cubes $L Q$ need not have bounded overlap if $L$ is large; this is indeed the case in our applications.

Lemma 4.16. Assume that $G \subset \mathbb{R}^{n}$ is an open set, $n \geq 2$. If $1<\kappa<\infty$ and $L \geq 1$, then, for every $g \in L^{\kappa}\left(\mathbb{R}^{n} \times \mathbb{R}^{n}\right)$,

$$
\sum_{Q \in \mathcal{W}(G)}|Q|^{2}\left(f_{L Q} f_{L Q}|g(x, y)| d y d x\right)^{\kappa} \leq C \int_{\mathbb{R}^{n}} \int_{\mathbb{R}^{n}}|g(x, y)|^{\kappa} d y d x
$$

where the constant $C>0$ only depends on $n$ and $\kappa$.

Proof. Throughout this proof, we write $m=2 n$. Recall that the (non-centered) maximal function (with respect to cubes) of a locally integrable function $f: \mathbb{R}^{m} \rightarrow$ $[-\infty, \infty]$ is

$$
\mathcal{M} f(x)=\sup _{x \in P} f_{P}|f(y)| d y
$$

where the supremum is taken over all cubes $P$ in $\mathbb{R}^{m}$ containing $x \in \mathbb{R}^{m}$. 
Rewriting the left-hand side of inequality (4.17) and using the definition of the maximal function in the point $(z, w) \in L Q \times L Q=P \subset \mathbb{R}^{m}$, we obtain

$$
\begin{aligned}
\sum_{Q \in \mathcal{W}(G)}|Q|^{2}\left(f_{L Q} f_{L Q}|g(x, y)| d y d x\right)^{\kappa} \\
\quad=\sum_{Q \in \mathcal{W}(G)} \int_{\mathbb{R}^{n}} \int_{\mathbb{R}^{n}} \chi_{Q}(z) \chi_{Q}(w)\left(f_{L Q} f_{L Q}|g(x, y)| d y d x\right)^{\kappa} d w d z \\
\leq \sum_{Q \in \mathcal{W}(G)} \int_{\mathbb{R}^{n}} \int_{\mathbb{R}^{n}} \chi_{Q}(z) \chi_{Q}(w)(\mathcal{M} g(z, w))^{\kappa} d w d z \\
\leq \int_{G} \int_{G}(\mathcal{M} g(z, w))^{\kappa} d w d z .
\end{aligned}
$$

The boundedness of the maximal operator $\mathcal{M}$ on $L^{\kappa}\left(\mathbb{R}^{m}\right)$ yields the claim.

We are now ready to prove our main result:

Proof of Theorem 4.1. Fix a number $q \in[1, p)$ such that $\beta=n(1 / p-1 / q)+s \in(0, s)$ and

$$
n-\lambda<\beta q<s p, \quad 0<1 / q-1 / p<\beta / n \text {. }
$$

Let $Q \in \mathcal{W}(G)$, and let $L \geq 1$ be as in Lemma 4.4. We first claim that

$$
\begin{aligned}
& \int_{Q}|u(x)|^{p} d x \leq C\left[\int_{Q}\left|u(x)-u_{Q}\right|^{p} d x+|Q|\left|u_{Q}\right|^{p}\right] \\
& \leq C|Q|^{1+p \beta / n-p / q}\left(\int_{L Q \cap G} \int_{L Q \cap G} \frac{|u(x)-u(y)|^{q}}{|x-y|^{n+\beta q}} d y d x\right)^{p / q} .
\end{aligned}
$$

For the term $\int_{Q}\left|u(x)-u_{Q}\right|^{p} d x$, the second inequality in (4.19) is a consequence of the $(p, q, \beta)$-Poincaré inequality of Lemma 4.3. On the other hand, for $\left|Q \| u_{Q}\right|^{p}$ the inequality follows from Lemma 4.4. Indeed, by the assumption (4.2) on the visual boundary and inequality (4.5), we have

$$
\begin{aligned}
\ell(Q)^{\lambda}\left|u_{Q}\right|^{q} & \leq C \mathcal{H}_{\infty}^{\lambda}\left(\partial_{x_{Q}, c}^{\mathrm{vis}} G\right)\left|u_{Q}\right|^{q} \\
& \leq C \ell(Q)^{\lambda-n+\beta q} \int_{L Q \cap G} \int_{L Q \cap G} \frac{|u(x)-u(y)|^{q}}{|x-y|^{n+\beta q}} d y d x
\end{aligned}
$$

and so

$$
|Q|\left|u_{Q}\right|^{p} \leq C|Q|^{1+p \beta / n-p / q}\left(\int_{L Q \cap G} \int_{L Q \cap G} \frac{|u(x)-u(y)|^{q}}{|x-y|^{n+\beta q}} d y d x\right)^{p / q}
$$

proving (4.19). 
Now property (2.1) of Whitney cubes and inequality (4.19) imply

$$
\begin{aligned}
& \int_{G} \frac{|u(x)|^{p}}{\operatorname{dist}(x, \partial G)^{s p}} d x \leq \sum_{Q \in \mathcal{W}(G)} \operatorname{diam}(Q)^{-s p} \int_{Q}|u(x)|^{p} d x \\
& \leq C \sum_{Q \in \mathcal{W}(G)}|Q|^{-s p / n}|Q|^{1+p \beta / n-p / q}\left(|Q|^{2} f_{L Q} f_{L Q} g(x, y) d y d x\right)^{p / q} \\
& \leq C \sum_{Q \in \mathcal{W}(G)}|Q|^{1+p \beta / n+p / q-s p / n}\left(f_{L Q} f_{L Q} g(x, y) d y d x\right)^{p / q},
\end{aligned}
$$

where we have written

$$
g(x, y)=\chi_{G}(x) \chi_{G}(y) \frac{|u(x)-u(y)|^{q}}{|x-y|^{n+\beta q}} .
$$

By the choice of $\beta$ we have in the last line of (4.20) that

$$
1+p \beta / n+p / q-s p / n=2,
$$

and thus Lemma 4.16, applied to the above function $g$ with $\kappa=p / q>1$, yields together with estimate (4.20) that

$$
\begin{aligned}
\int_{G} \frac{|u(x)|^{p}}{\operatorname{dist}(x, \partial G)^{s p}} d x & \leq C \int_{\mathbb{R}^{n}} \int_{\mathbb{R}^{n}} g(x, y)^{p / q} d y d x \\
& \leq C \int_{G} \int_{G} \frac{|u(x)-u(y)|^{p}}{|x-y|^{n+s p}} d x d y .
\end{aligned}
$$

Note that we used above also the identity $p(n+\beta q) / q=n+s p$ to obtain the correct exponent in the denominator.

The following corollary is a consequence of Theorem 4.1 and Proposition 3.3.

Corollary 4.21. Let $0<s<1$ and $1<p<\infty$ satisfy $0<s p<n$. Assume that $G \subset \mathbb{R}^{n}$ is a uniform domain and that there exists $n-s p<\lambda \leq n$ and $C>0$ such that

$$
\mathcal{H}_{\infty}^{\lambda}(B(x, 2 \operatorname{dist}(x, \partial G)) \cap \partial G) \geq C \operatorname{dist}(x, \partial G)^{\lambda}
$$

for all $x \in G$. Then $G$ admits an $(s, p)$-Hardy inequality.

Observe that Corollary 1.4, stated in the Introduction, is now a consequence of Corollary 4.21 and Proposition 3.7. We also refer to the discussion in the beginning of Section 3.

We conclude this section with a partial relaxation of the visual boundary condition of Theorem 4.1. The following modification of Lemma 4.4 shows that we do not need to assume the visibility of the boundary with respect to cubes $Q \in \mathcal{W}(G)$ if the nearby complement is of zero measure.

Lemma 4.23. Let $G \subset \mathbb{R}^{n}$ be an open set and assume that $0<\beta<1,1 \leq q<\infty$, and $n-\beta q<\lambda \leq n$. Fix $c \geq 1$ and let $Q \in \mathcal{W}(G)$. Assume that $L>1$ and $\left|G^{c} \cap L Q\right|=0$. Then there exists $C=C(n, c, \lambda, q, \beta, L)>0$, independent of $Q$, such that

$$
\mathcal{H}_{\infty}^{\lambda}(\partial G \cap L Q)\left|u_{Q}\right|^{q} \leq C \ell(Q)^{\lambda-n+\beta q} \int_{L Q \cap G} \int_{L Q \cap G} \frac{|u(x)-u(y)|^{q}}{|x-y|^{n+\beta q}} d y d x
$$

for every $u \in C_{0}^{\infty}(G)$. 
Proof. Let us indicate here the main differences compared to the proof of Lemma 4.4. For $w \in \partial G \cap L Q$ we can now consider, instead of the John-type chain $C(Q, w)$, a chain $\widetilde{C}(Q, w)=\left\{Q_{0}, Q_{1}, \ldots\right\}$ of cubes, where $Q_{0}=Q, Q_{1}=L Q, w \in Q_{i+1} \subset Q_{i}$ for every $i \geq 1$, and $\ell\left(Q_{i+1}\right)=\ell\left(Q_{i}\right) / 2$ for every $i \geq 1$. Estimate (4.12) (but with the integrals taken over $\left.Q_{i}\right)$ is a simple consequence of the $(1, q, \beta)$-Poincaré inequality for cubes, and thus we find from the chain $\widetilde{C}(Q, w)$ a cube $Q_{w}$ as in (4.13) (note that here the constant depends on $L$ ). We can now choose disjoint cubes $Q_{j}=Q_{w_{j}}$ such that the dilated cubes $5 Q_{j}$ cover the set $\partial G \cap L Q$, and thus we obtain, just like in the proof of Lemma 4.4, that

$$
\begin{aligned}
\mathcal{H}_{\infty}^{\lambda}(\partial G \cap L Q) & \leq C \sum_{j=1}^{N} \ell\left(Q_{j}\right)^{\lambda} \\
& \leq C \sum_{j=1}^{N} \ell(Q)^{\lambda-n+\beta q} \int_{Q_{j}} \int_{Q_{j}} \frac{|u(x)-u(y)|^{q}}{|x-y|^{n+\beta q}} d y d x \\
& \leq C \sum_{j=1}^{N} \ell(Q)^{\lambda-n+\beta q} \int_{Q_{j}} \int_{L Q} \frac{|u(x)-u(y)|^{q}}{|x-y|^{n+\beta q}} d y d x \\
& \leq C \ell(Q)^{\lambda-n+\beta q} \int_{L Q} \int_{L Q} \frac{|u(x)-u(y)|^{q}}{|x-y|^{n+\beta q}} d y d x \\
& \leq C \ell(Q)^{\lambda-n+\beta q} \int_{L Q \cap G} \int_{L Q \cap G} \frac{|u(x)-u(y)|^{q}}{|x-y|^{n+\beta q}} d y d x .
\end{aligned}
$$

The last line above follows from the assumption $\left|G^{c} \cap L Q\right|=0$.

Remark 4.26. Let $G \subset \mathbb{R}^{n}$ be an open set and assume that there exists $0<\lambda \leq n$, $c \geq 1, L \geq 1$, and $C>0$ such that for every $Q \in \mathcal{W}(G)$ (at least) one of the following conditions holds:

(a) $\mathcal{H}_{\infty}^{\lambda}\left(\partial_{x_{Q}, c}^{\mathrm{vis}} G\right) \geq C \ell(Q)^{\lambda}$; or,

(b) $\left|G^{c} \cap L Q\right|=0$ and $\mathcal{H}_{\infty}^{\lambda}(\partial G \cap L Q) \geq C \ell(Q)^{\lambda}$.

Then we can use either Lemma 4.4 or Lemma 4.23 to yield estimate (4.19) in the proof of Theorem 4.1, and we conclude that such an open set $G$ admits $(s, p)$-Hardy inequalities for all $1<p<\infty$ and $0<s<1$ satisfying $n-\lambda<s p<n$.

Note that in the case $\left|G^{c}\right|=0$, where we can use the above case (b) for every cube $Q \in \mathcal{W}(G)$, the same conclusion also follows from Theorem 3.4 and Theorem 6.5 below.

\section{Counterexamples in the Plane}

In this section, we show that $(s, p)$-uniform fatness of the boundary $\partial G$ is not sufficient for $G$ to admit an $(s, p)$-Hardy inequality. Similar examples exist in higher dimensions as well, but, for the sake of clarity, we confine ourselves here to the planar case. Let us formulate this as a theorem:

Theorem 5.1. Let $1<p<\infty$ and $0<s<1$ be such that $0<s p \leq 1$. Then there exists an open set $G \subset \mathbb{R}^{2}$ whose boundary is $(s, p)$-uniformly fat, but which does not admit $(s, p)$-Hardy inequalities. In addition, there exists a bounded domain $G^{\prime} \subset \mathbb{R}^{2}$, with a locally $(s, p)$-uniformly fat boundary, such that $G^{\prime}$ does not admit $(s, p)$-Hardy inequalities. 
We base our constructions on the examples in [5], but we need significant modifications in order to adapt them for our purposes. To clarify this, let $1<p<\infty$ and $0<s<1$ be such that $s p \leq 1$. Then the domain

$$
G^{\text {core }}:=(-1,1)^{2} \subset \mathbb{R}^{2}
$$

does not admit an $(s, p)$-Hardy inequality, we refer to [5] or the proof of Proposition 5.2. Albeit the complement of $G^{\text {core }}$ is $(s, p)$-uniformly fat, this counterexample does not suffice for the proof of Theorem 5.1, as the boundary of $G^{\text {core }}$ is still too 'thin'; it is $(s, p)$-uniformly fat only for $s p>1$, exactly the same range for which the set $G^{\text {core }}$ admits $(s, p)$-Hardy inequalities.

We give two possible ways to address this problem: In the first one, given in Sections 5.1-5.4, we place a fat Cantor set - one with positive measure - to each of the Whitney cubes in $\mathcal{W}\left(\mathbb{R}^{2} \backslash \bar{G}^{\text {core }}\right)$. This is done in a quantitative manner, ensuring that $(s, p)$-Hardy inequalities still remain false. The construction yields an open set $G$ whose boundary is an unbounded 2-regular set, and consequently, by Proposition 3.15 , the boundary $\partial G$ is indeed $(s, p)$-uniformly fat, thus proving the first claim in Theorem 5.1. The second claim is proved in Sections 5.5-5.7. There we consider localized examples, and the resulting sets $G$ are even domains. However, in these latter examples much more care must be taken in the choices of the test functions and in the related calculations.

5.1. Outline of the first example. The construction consists of two steps. In the first step we construct a John domain $G^{\text {core }}$ satisfying the following conditions (A) $-(\mathrm{D})$ :

(A) there is a constant $C>0$ such that for any $m \in \mathbb{N}$, the boundary $\partial G^{\text {core }}$ can be covered by using at most $C m^{2-s p}$ balls in the family $\{B(x, 1 /(2 m)): x \in$ $\left.\partial G^{\text {core }}\right\}$

(B) $\int_{G^{\mathrm{core}}} \operatorname{dist}\left(x, \partial G^{\mathrm{core}}\right)^{-s p} d x=\infty$;

(C) $\partial G^{\text {core }}=\partial\left(\mathbb{R}^{2} \backslash \bar{G}^{\text {core }}\right)$ and $\left|\partial G^{\text {core }}\right|=0$;

(D) the following 'co-plumpness' condition is satisfied for some $\eta \in(0,1)$ : for each $x \in \mathbb{R}^{2} \backslash G^{\text {core }}$ and each $r>2 \operatorname{dist}\left(x, \partial G^{\text {core }}\right)$, there is $Q \in \mathcal{W}\left(\mathbb{R}^{2} \backslash \bar{G}^{\text {core }}\right)$ such that $|Q| \geq \eta r^{2}$ and $Q \subset B(x, r)$.

Conditions (A) and (B) are taken from [5], and they imply that $G^{\text {core }}$ does not admit an $(s, p)$-Hardy inequality. The two other conditions (C) and (D) are technical, required in the second step of the construction. In the case of $s p=1$, we may begin our construction with $G^{\text {core }}:=(-1,1)^{2}$. For the remaining cases $0<s p<1$, we refer to Section 5.2.

In the second step, we construct an open set $G \subset \mathbb{R}^{2}$ with the following properties:

(1) $G^{\text {core }} \subset G$;

(2) $\partial G^{\text {core }} \subset \partial G$;

(3) $\sum_{j=-\infty}^{\infty} 2^{j s p} \sum_{Q \in \mathcal{W}_{j}\left(\mathbb{R}^{2} \backslash \bar{G}^{\text {core }}\right)}|Q \cap G|<\infty$;

(4) $\partial G$ is $(s, p)$-uniformly fat.

The construction of such sets is given in Section 5.4. Let us now show that a set $G$ satisfying (1)-(4) indeed gives a desired counterexample:

Proposition 5.2. Suppose that an open set $G$ satisfies conditions (1)-(3) above. Then $G$ does not admit an $(s, p)$-Hardy inequality. 
Proof. We use test functions $u_{m} \in C_{0}^{\infty}\left(G^{\text {core }}\right)$ with $m \geq 1$ large enough. These are defined by

$$
u_{m}:=\varphi_{8 m} * \chi_{\left\{x \in G^{\text {core }}: \operatorname{dist}\left(x, \partial G^{\text {core }}\right) \geq 3 /(8 m)\right\}},
$$

where $\varphi_{m}=m^{2} \varphi(m x)$ and $\varphi \in C_{0}^{\infty}(B(0,1))$ is a non-negative bump function, with $\int \varphi d x=1$. Some of the computations in [5] are invoked in the sequel, and actually the only properties we explicitly need for the functions $u_{m}$ are that $0 \leq u_{m} \leq 1$ for all $m$, and that they converge pointwise to $\chi_{G^{\text {core }}}$ monotonically, i.e. $u_{m+1} \geq u_{m}$ for all $m$.

By conditions (1), (2), and (B),

$$
\int_{G} \frac{\left|u_{m}(x)\right|^{p}}{\operatorname{dist}(x, \partial G)^{s p}} d x \geq \int_{G^{\text {core }}} \frac{\left|u_{m}(x)\right|^{p}}{\operatorname{dist}\left(x, \partial G^{\text {core }}\right)^{s p}} d x \stackrel{m \rightarrow \infty}{\longrightarrow} \infty .
$$

Hence, it suffices to show that the following integrals are uniformly bounded in $m$ :

$$
\begin{aligned}
& \int_{G} \int_{G} \frac{\left|u_{m}(x)-u_{m}(y)\right|^{p}}{|x-y|^{2+s p}} d y d x \\
& =\left\{\int_{G^{\text {core }}} \int_{G^{\text {core }}}+2 \int_{G \backslash G^{\text {core }}} \int_{G^{\text {core }}}\right\} \frac{\left|u_{m}(x)-u_{m}(y)\right|^{p}}{|x-y|^{2+s p}} d y d x .
\end{aligned}
$$

The integral over the set $G^{\text {core }} \times G^{\text {core }}$ is uniformly bounded in $m$; we refer to $[5$, pp. 577-578] for a computation which relies on the covering property (A). Hence, it suffices to estimate the second integral,

$$
\begin{aligned}
S_{m}: & =\int_{G \backslash G^{\mathrm{core}}} \int_{G^{\mathrm{core}}} \frac{\left|u_{m}(x)-u_{m}(y)\right|^{p}}{|x-y|^{2+s p}} d y d x \\
& =\sum_{j=-\infty}^{\infty} \sum_{Q \in \mathcal{W}_{j}\left(\mathbb{R}^{2} \backslash \bar{G}^{\text {core }}\right)} \int_{G \cap Q} \int_{G^{\mathrm{core}}} \frac{\left|u_{m}(y)\right|^{p}}{|x-y|^{2+s p}} d y d x ;
\end{aligned}
$$

we used above condition $(\mathrm{C})$, which assures that $\partial G^{\text {core }}$ has zero measure. Again, by condition (C), for every $x \in G \cap Q \subset \mathbb{R}^{2} \backslash \bar{G}^{\text {core }}$ (with $Q$ as in the summation above) and $y \in G^{\text {core }}$,

$$
|x-y| \geq \operatorname{dist}\left(x, \partial G^{\text {core }}\right)=\operatorname{dist}\left(x, \partial\left(\mathbb{R}^{2} \backslash \bar{G}^{\text {core }}\right)\right) \geq \operatorname{diam}(Q) \geq 2^{-j} .
$$

Since the test functions $u_{m}$ are bounded by 1 , we may integrate in polar coordinates in order to see that

$$
\begin{aligned}
S_{m} & \leq \sum_{j=-\infty}^{\infty} \sum_{Q \in \mathcal{W}_{j}\left(\mathbb{R}^{2} \backslash \bar{G}^{\text {core })}\right.} \int_{G \cap Q} \int_{\mathbb{R}^{2} \backslash B\left(x, 2^{-j}\right)} \frac{1}{|x-y|^{2+s p}} d y d x \\
& \lesssim \sum_{j=-\infty}^{\infty} 2^{j s p} \sum_{Q \in \mathcal{W}_{j}\left(\mathbb{R}^{2} \backslash \bar{G}^{\text {core }}\right)}|G \cap Q|<\infty .
\end{aligned}
$$

The last step follows from condition (3).

5.2. Construction of $G^{\text {core }}$. Let $1<p<\infty$ and $0<s<1$ be such that $s p<$ 1. We construct a domain satisfying properties (A)-(D) from the beginning of Section 5.1. Let $\lambda=2-s p$ and let $G^{\text {core }}$ be a snowflake domain, which is a coplump John domain whose boundary $E:=\partial G^{\text {core }}$ is a $\lambda$-regular set and satisfies $E=\partial\left(\mathbb{R}^{2} \backslash \bar{G}^{\text {core }}\right)$. The usual von Koch snowflake, illustrated in Figure 1, has dimension $\lambda=\log 4 / \log 3$, but the construction can be modified to obtain a set $E$ 
of any dimension $\lambda \in(1,2)$; see for instance [16, Sect. 2]. In particular, $E$ has zero Lebesgue measure. Thus, conditions (C) and (D) are satisfied by the construction

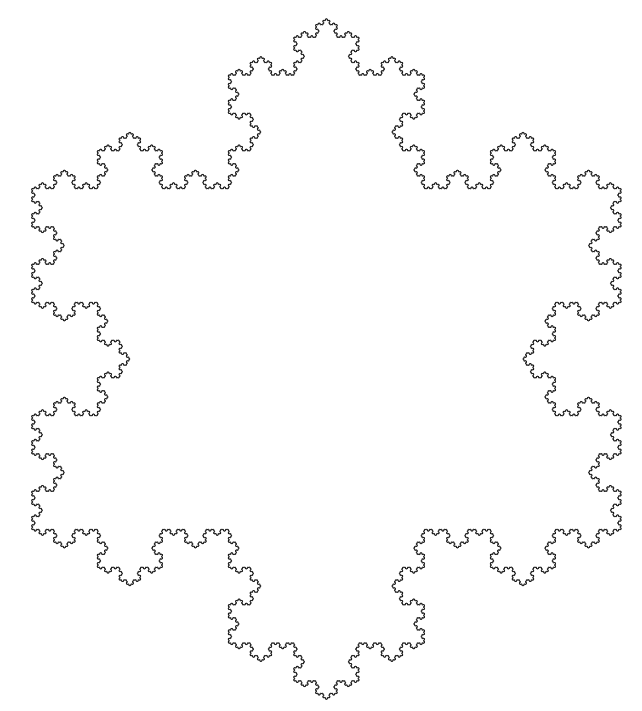

Figure 1. The usual von Koch snowflake.

In order to verify the remaining two conditions (A) and (B), we use ideas from [20, Lemma 2.1]. Fix $0<r \leq 1 / 5$ and consider the family $\mathcal{F}_{r}=\{B(x, r): x \in E\}$. By the $5 r$-covering lemma, there are pairwise disjoint balls $B_{1}^{r}, \ldots, B_{N_{r}}^{r} \in \mathcal{F}_{r}$ such that $E \subset \cup_{i=1}^{N_{r}} 5 B_{i}^{r}$. By the $\lambda$-regularity condition (2.3) and the pairwise disjointedness of the balls $B_{i}^{r}$, we have

$$
\begin{aligned}
\mathcal{H}^{\lambda}(E) & \leq \sum_{i=1}^{N_{r}} \mathcal{H}^{\lambda}\left(5 B_{i}^{r} \cap E\right) \leq C 5^{\lambda} N_{r} r^{\lambda} \\
& \leq C^{2} 5^{\lambda} \sum_{i=1}^{N_{r}} \mathcal{H}^{\lambda}\left(B_{i}^{r} \cap E\right) \leq C^{2} 5^{\lambda} \mathcal{H}^{\lambda}(E),
\end{aligned}
$$

and, hence, $C_{1} r^{-\lambda} \leq N_{r} \leq C_{2} r^{-\lambda}$, where the positive constants $C_{1}$ and $C_{2}$ do not depend on $r$, in particular $N_{r} \simeq r^{-\lambda}$. As a consequence, we obtain the covering property (A) for $G^{\text {core }}$.

It remains to verify condition (B). By denoting

$$
E_{r}=\left\{x \in G^{\text {core }}: \operatorname{dist}(x, E) \leq r\right\},
$$

we have

$$
\begin{aligned}
& \int_{G^{\mathrm{core}}} \operatorname{dist}(x, E)^{\lambda-2} d x \\
& =\int_{0}^{\infty}\left|\left\{x \in G^{\text {core }}: \operatorname{dist}(x, E)^{\lambda-2} \geq t\right\}\right| d t=\int_{0}^{\infty}\left|E_{t^{1 /(\lambda-2)}}\right| d t .
\end{aligned}
$$

Thus, for the last condition, it suffices to show that, for some $C>0$ independent of $r$,

$$
\left|E_{r}\right| \geq C r^{2-\lambda}, \quad r \in(0,1 / 5) .
$$


We can deduce this estimate as follows. Let us first observe that, by John property of $G^{\text {core }}$, there is a constant $C$ such that $\left|B_{i}^{r} \cap G^{\text {core }}\right| \geq C\left|B_{i}^{r}\right|$. Hence, by the pairwise disjointedness of the balls $B_{i}^{r}$,

$$
\begin{aligned}
\left|E_{r}\right| & \geq\left|\bigcup_{i=1}^{N_{r}} B_{i}^{r} \cap G^{\mathrm{core}}\right|=\sum_{i=1}^{N_{r}}\left|B_{i}^{r} \cap G^{\mathrm{core}}\right| \\
& \geq C \sum_{i=1}^{N_{r}}\left|B_{i}^{r}\right|=C \sum_{i=1}^{N_{r}} r^{2}=C N_{r} r^{2} \geq C r^{2-\lambda},
\end{aligned}
$$

and we conclude that the property (B) holds, i.e. $\int_{G^{\text {core }}} \operatorname{dist}(x, E)^{-s p} d x=\infty$.

5.3. Fat Cantor sets. We construct next an auxiliary compact set $K_{j}=K_{j}\left(Q_{j}\right) \subset$ $Q_{j}$. Here $Q_{j} \subset \mathbb{R}^{2}$ is a given closed cube with $\ell\left(Q_{j}\right)=2^{-j}, j \in \mathbb{Z}$, and the construction is parameterized by a given sequence $\left\{\varepsilon_{j, k}\right\}_{k \geq 0}$ of real numbers in $(0,1 / 2)$ such that $\sum_{k=0}^{\infty} \varepsilon_{j, k}<1 / 2$.

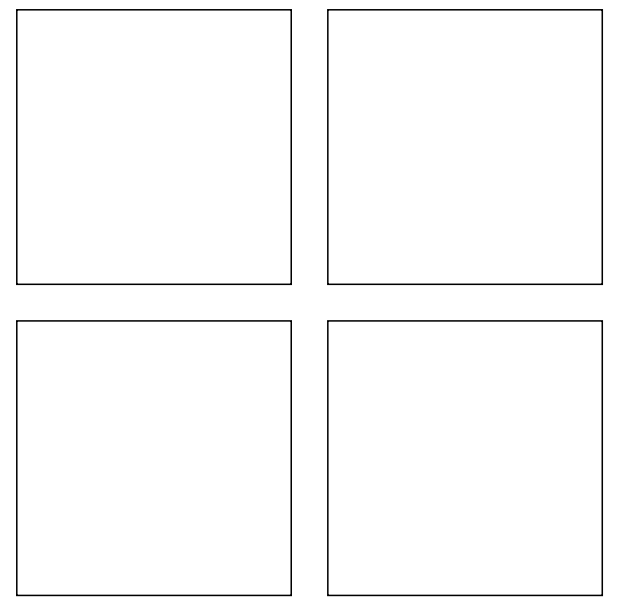

FiguRE 2. We have removed two rectangles from a closed cube $Q_{j, k, m}$. The four closed sub cubes belong to generation $k+1$, and they are denoted by $Q_{j, k+1, m_{1}}, \ldots, Q_{j, k+1, m_{4}}$ for some $\left\{m_{1}, \ldots, m_{4}\right\} \in$ $\left\{1,2, \ldots, 4^{k+1}\right\}$.

We construct cubes $Q_{j, k, m}$ with common side length $\ell_{j, k}$, where $m \in\left\{1, \ldots 4^{k}\right\}$ is used to index the cubes of generation $k \geq 0$. Let $Q_{j, 0,1}=Q_{j}$ and define $\ell_{j, 0}=$ $2^{-j}$. At the inductive stage, we are given $k \geq 0$ and the cubes $Q_{j, k, m}$ whose side lengths are $\ell\left(Q_{j, k, m}\right)=\ell_{j, k}, m=1, \ldots, 4^{k}$. Each of these cubes is partitioned in five sets: to the union of two overlapping perpendicular rectangles, both similar to $\left[0, \ell_{j, k}\right] \times\left(0, \varepsilon_{j, k} \ell_{j, k}\right)$ and having midpoint $x_{Q_{j, k, m}}$; and to four similar closed cubes of side length $\ell_{j, k+1}=\left(1-\varepsilon_{j, k}\right) \ell_{j, k} / 2$. The resulting $4^{k+1}$ 'generation $k+1$ ' cubes are denoted by $Q_{j, k+1, m}, m=1, \ldots, 4^{k+1}$; i.e., we fix an ordering for these cubes. For an illustration, we refer to Figure 2. It is immediate from the construction that

$$
\ell_{j, k} / 4 \leq \ell_{j, k+1} \leq \ell_{j, k} / 2 \text { and } \ell_{j, k^{\prime}} \leq 2^{k-k^{\prime}} \ell_{j, k} \text { if } k^{\prime} \geq k \text {. }
$$

We define compact sets $K_{j, 0} \supset K_{j, 1} \supset \cdots \supset K_{j}$ by setting

$$
K_{j, k}:=\bigcup_{m=1}^{4^{k}} Q_{j, k, m}, \quad K_{j}:=\bigcap_{k \geq 0} K_{j, k} .
$$


The following 'quantitative Lebesgue density theorem' for $K_{j}$ will be useful in several occasions.

Lemma 5.5. Suppose that $k \geq 0$ and $m \in\left\{1,2, \ldots, 4^{k}\right\}$ are fixed. Then

$$
\left|K_{j} \cap Q_{j, k, m}\right| \geq\left|Q_{j, k, m}\right|\left(1-\sum_{k^{\prime}=k}^{\infty} 2 \varepsilon_{j, k^{\prime}}\right) .
$$

Proof. Since $\left|K_{j} \cap Q_{j, k, m}\right|=\lim _{k^{\prime} \rightarrow \infty}\left|K_{j, k^{\prime}} \cap Q_{j, k, m}\right|$, it suffices to prove the inequality

$$
\left|K_{j, k^{\prime}} \cap Q_{j, k, m}\right| \geq\left|Q_{j, k, m}\right|\left(1-\sum_{k^{\prime \prime}=k}^{k^{\prime}-1} 2 \varepsilon_{j, k^{\prime \prime}}\right), \quad k^{\prime} \geq 0 .
$$

Let us observe that $K_{j, k^{\prime}} \cap Q_{j, k, m} \supset K_{j, k} \cap Q_{j, k, m}=Q_{j, k, m}$ for $0 \leq k^{\prime} \leq k$; hence, inequality (5.7) holds for these values of $k^{\prime}$. In the inductive stage, we assume (5.7) for some $k^{\prime} \geq k$. Then, by construction, the intersection $K_{j, k^{\prime}} \cap Q_{j, k, m}$ is a union of $4^{k^{\prime}-k}$ cubes, each of which produces four 'generation $k^{\prime}+1$ ' cubes after removal of a set (which is a union of two perpendicular rectangles) whose area is strictly bounded by

$$
2 \varepsilon_{j, k^{\prime}} \ell_{j, k^{\prime}}^{2} \leq 2 \varepsilon_{j, k^{\prime}}\left|Q_{j, k, m}\right| 4^{k-k^{\prime}}
$$

In the last step above, we applied (5.4). By the induction assumption, the area of $K_{j, k^{\prime}+1} \cap Q_{j, k, m}$ is bounded from below by

$$
\left|K_{j, k^{\prime}} \cap Q_{j, k, m}\right|-4^{k^{\prime}-k} 2 \varepsilon_{j, k^{\prime}}\left|Q_{j, k, m}\right| 4^{k-k^{\prime}} \geq\left|Q_{j, k, m}\right|\left(1-\sum_{k^{\prime \prime}=k}^{\left(k^{\prime}+1\right)-1} 2 \varepsilon_{j, k^{\prime \prime}}\right) .
$$

The proof of inequality (5.7) is complete.

Remark 5.8. A consequence of Lemma 5.5 is the following. For every $x \in K_{j}$ and every radius $0<r \leq 10 \sqrt{2} \cdot 2^{-j}$,

$$
\left|B(x, r) \cap K_{j}\right| \geq r^{2}(10 \sqrt{2})^{-2}\left(1-\sum_{k^{\prime}=0}^{\infty} 2 \varepsilon_{j, k^{\prime}}\right) .
$$

(The factor $10 \sqrt{2}$ is not special, but chosen for our convenience.) In order to verify this, we choose $k \geq 0$ such that $\ell_{j, k} / 4<r /(10 \sqrt{2}) \leq \ell_{j, k}$. This is possible by inequalities (5.4). Then we fix an index $m$ in such a way that $x \in Q_{j, k, m}$. Now $Q_{j, k, m} \subset B(x, r)$, and the claim follows from inequality (5.6) by obvious estimates.

5.4. The construction of $G$. Finally, we construct an open set $G$ with properties (1)-(4) stated in Section 5.1. Recall that we have at our disposal a (bounded) John domain $G^{\text {core }}$ satisfying conditions $(\mathrm{A})-(\mathrm{D})$ stated in Section 5.1.

We choose a sequence $\left\{\varepsilon_{j}\right\}_{j \in \mathbb{Z}}, \varepsilon_{j} \in(0,1 / 2)$, such that

$$
\sum_{j=-\infty}^{\infty} \varepsilon_{j} 2^{j s p} \sum_{Q \in \mathcal{W}_{j}\left(\mathbb{R}^{2} \backslash \bar{G}^{\text {core }}\right)}|Q|<\infty .
$$

This is possible since $G^{\text {core }}$ is bounded, and hence the inner sum is finite for each $j$. Then, we let $\varepsilon_{j, k}:=\varepsilon_{j} 2^{-k} / 4$ for each $j \in \mathbb{Z}$ and $k \geq 0$. Observe, in particular, that

$$
\sum_{k^{\prime}=0}^{\infty} \varepsilon_{j, k^{\prime}}=\varepsilon_{j} / 2<1 / 4, \quad j \in \mathbb{Z}
$$


Define a closed set that is parameterized by $\left\{\varepsilon_{j, k}\right\}$ and has no interior points,

$$
K:=\partial G^{\text {core }} \cup \bigcup\left\{x: x \in K_{j}(Q) \text { for some } Q \in \mathcal{W}_{j}\left(\mathbb{R}^{2} \backslash \bar{G}^{\text {core }}\right) \text { and } j \in \mathbb{Z}\right\} .
$$

Here the compact sets $K_{j}(Q)$ are as defined in Section 5.3. Observe that it is important to include the boundary of $G^{\text {core }}$ to the union above, as otherwise $K$ would not be closed. Finally, we define the desired open set to be $G:=\mathbb{R}^{2} \backslash K$. Note that $\partial G=K$ since the closed set $K$ does not contain any interior points.

The proof of the first claim in Theorem 5.1 is finished by Proposition 5.2 and the following result.

Proposition 5.11. The open set $G=\mathbb{R}^{2} \backslash K$ satisfies conditions (1)-(4).

Proof. By construction, conditions (1) and (2) are satisfied. Let us then consider condition (3). Observe that $|Q \cap G|=\left|Q \backslash K_{j}(Q)\right|$ if $Q \in \mathcal{W}_{j}\left(\mathbb{R}^{2} \backslash \bar{G}^{\text {core }}\right)$. Hence, by Lemma 5.5 (recall that $Q_{j, 0,1}=Q_{j}=Q$ therein),

$$
\sum_{j=-\infty}^{\infty} 2^{j s p} \sum_{Q \in \mathcal{W}_{j}\left(\mathbb{R}^{2} \backslash \bar{G}^{\text {core }}\right)}|Q \cap G| \leq \sum_{j=-\infty}^{\infty} 2^{j s p} \sum_{Q \in \mathcal{W}_{j}\left(\mathbb{R}^{2} \backslash \bar{G}^{\text {core }}\right)}|Q| \sum_{k^{\prime}=0}^{\infty} 2 \varepsilon_{j, k^{\prime}} .
$$

Condition (3) follows from this by using inequalities (5.10) and (5.9). In order to verify condition (4), it suffices to show that $\partial G=K$ is an unbounded 2-regular set. Indeed, Proposition 3.15 then implies that $\partial G$ is $(s, p)$-uniformly fat.

For a fixed $x \in K$ and $0<r<\infty$, we have either (a) or (b) below:

(a): $\operatorname{dist}\left(x, \partial G^{\text {core }}\right)<r / 2$. By co-plumpness, condition (D), there is $Q \in \mathcal{W}\left(\mathbb{R}^{2} \backslash\right.$ $\left.\bar{G}^{\text {core }}\right)$ such that $|Q| \geq \eta r^{2}$ and $Q \subset B(x, r)$. By Lemma 5.5 and inequality (5.10),

$$
|B(x, r) \cap K| \geq\left|K_{j}(Q)\right| \geq r^{2} \eta / 2 .
$$

(b): $r / 2 \leq \operatorname{dist}\left(x, \partial G^{\text {core }}\right)$. In this case, $x \in K_{j}(Q) \subset Q$ for $Q \in \mathcal{W}_{j}\left(\mathbb{R}^{2} \backslash \bar{G}^{\text {core }}\right)$, $j \in \mathbb{Z}$. Thus, by condition (C) and properties of Whitney cubes,

$$
r / 2 \leq \operatorname{dist}\left(x, \partial\left(\mathbb{R}^{2} \backslash \bar{G}^{\text {core }}\right)\right) \leq 5 \operatorname{diam}(Q)=5 \sqrt{2} \cdot 2^{-j} .
$$

As a conclusion $r \leq 10 \sqrt{2} \cdot 2^{-j}$, and so, by Remark 5.8 and inequality (5.10),

$$
|B(x, r) \cap K| \geq\left|B(x, r) \cap K_{j}(Q)\right| \geq r^{2}(10 \sqrt{2})^{-2} / 2 .
$$

This concludes the proof of condition (4).

5.5. A local construction. A drawback in the open set $G=\mathbb{R}^{2} \backslash K$ of the previous construction is that $G$ is not connected. In the following Subsections 5.6 and 5.7, we indicate alternative counterexamples where $G$ is indeed a domain, thus showing that the non-connectivity is not an issue in the failure of the $(s, p)$-Hardy inequalities in the construction of Section 5.1. The examples in Sections 5.6 and 5.7 are based on the use of 'localized' test functions constructed in this subsection. Again, the examples are given only for the planar case, but similar constructions can be carried out, for $0<s p \leq 1$ (corresponding to $n-1 \leq \lambda<n$ ), in higher dimensions as well.

For $1 \leq \lambda<2$, let $K_{\lambda} \subset\left\{\left(x_{1}, x_{2}\right) \in \mathbb{R}^{2}: x_{2} \geq 0\right\}$ be a $\lambda$-dimensional von Koch -type snowflake curve joining the points $(-3,0)$ and $(3,0)$ in the plane. For instance, when $\lambda=1$, we simply have the interval from $(-3,0)$ to $(3,0)$, and the von Koch snowflake curve in Figure 1 is obtained by joining together three copies of the curve $K_{\lambda}$, with $\lambda=\log 4 / \log 3$, to create a closed curve. 
Let $G \subset \mathbb{R}^{2}$ be any domain such that

$$
K_{\lambda}=\partial G \cap([-3,3] \times[0,3])
$$

and $[-3,3] \times[0,-1] \subset G^{c}$; note that by the connectivity of $G$, then also the set bounded by $K_{\lambda}$ and the interval from $(-3,0)$ to $(3,0)$ belongs to $G^{c}$. We demonstrate below that such a domain $G$ can not admit $(s, p)$-Hardy inequalities when $s p=2-\lambda$. This is not yet enough to produce the desired counterexamples, but in Sections 5.6 and 5.7 we explain how to modify these domains so that we obtain a proof for the second claim in Theorem 5.1. Nevertheless, the construction below has also independent interest. In particular, when $G$ is chosen to be the upper half-space, with $\lambda=1$, we see that the case (T3) of Theorem 1.1 in [5] is sharp: $G$ is a domain above the graph of a Lipschitz function $\mathbb{R}^{n-1} \rightarrow \mathbb{R}$, and $G$ does not admit $(s, p)$-Hardy inequalities when $s p=1$.

For $j, m \in \mathbb{N}$, we define

$$
\begin{aligned}
& N_{j}=\left\{x \in[-1,1] \times[0,2]: 2^{-j} \leq \operatorname{dist}(x, \partial G)<2^{-j+1}\right\}, \\
& A_{m}=\left\{x \in[-1,1] \times[0,2]: 2^{-m}<\operatorname{dist}(x, \partial G) \leq 1\right\}=\bigcup_{j=1}^{m} N_{j}, \\
& E_{m}=\left\{x \in[-2,2] \times[0,2]: 0<\operatorname{dist}(x, \partial G) \leq 2^{-m}\right\} .
\end{aligned}
$$

We choose functions $u_{m} \in C_{0}^{\infty}(G)$ such that:

(1) $u_{m}=1$ in $A_{m}$;

(2) $u_{m}=0$ outside $[-2,2] \times[0,2]$;

(3) $0 \leq u_{m} \leq 1$ everywhere;

(4) $\left|\nabla u_{m}\right| \leq c 2^{m}$ everywhere and $\left|\nabla u_{m}\right| \leq c$ in $G \backslash E_{m}$.

Let $1<p<\infty$ and $0<s<1$ be such that $s p=2-\lambda$. Since $u_{m}=1$ in $A_{m}$ and $\left|N_{j}\right| \simeq 2^{j(\lambda-2)}$ by the $\lambda$-regularity of $K_{\lambda}$, it follows that

$$
\int_{G} \frac{\left|u_{m}(x)\right|^{p}}{\operatorname{dist}(x, \partial G)^{s p}} d x \geq \sum_{j=1}^{m} \int_{N_{j}} \frac{1}{\operatorname{dist}(x, \partial G)^{s p}} d x \simeq \sum_{j=1}^{m} 2^{j(\lambda-2)} 2^{j s p}=m .
$$

Hence, it suffices to show that the right-hand side of the $(s, p)$-Hardy inequality remains uniformly bounded in $m$. We do this using arguments similar to those in Dyda's example [5], but due to the local nature of our example, we have additional terms to estimate in our calculations. First, we observe that

$$
\begin{aligned}
\int_{G} \int_{G} \frac{\left|u_{m}(x)-u_{m}(y)\right|^{p}}{|x-y|^{2+s p}} d x d y \\
\quad \leq\left\{2 \int_{G} \int_{E_{m}}+\int_{G \backslash E_{m}} \int_{G \backslash E_{m}}\right\} \frac{\left|u_{m}(x)-u_{m}(y)\right|^{p}}{|x-y|^{2+s p}} d x d y=: 2 I_{1}+I_{2} .
\end{aligned}
$$


By the $\lambda$-regularity of the snowflake curves, it is easy to see that $E_{m} \subset \bigcup_{k=1}^{N_{m}} B_{k}$, where $B_{k}=B\left(x_{k}, 2^{-m+1}\right)$ for some $x_{k} \in K_{\lambda}$, and $N=N_{m} \leq c 2^{\lambda m}$. Thus, we obtain

$$
\begin{aligned}
I_{1} & \leq c \sum_{k=1}^{N} \int_{G} \int_{B_{k}} \frac{\left|u_{m}(x)-u_{m}(y)\right|^{p}}{|x-y|^{2+s p}} d x d y \\
& \leq c \sum_{\ell=0}^{\infty} \sum_{k=1}^{N} \int_{(\ell+1) B_{k} \backslash \ell B_{k}} \int_{B_{k}} \frac{\left|u_{m}(x)-u_{m}(y)\right|^{p}}{|x-y|^{2+s p}} d x d y .
\end{aligned}
$$

Using property (4) of the functions $u_{m}$, the terms for $\ell=0$ and $\ell=1$ in the above sum are bounded by

$$
\begin{gathered}
\sum_{k=1}^{N} \int_{2 B_{k}} \int_{B_{k}} \frac{2^{m p}|x-y|^{p}}{|x-y|^{2+s p}} d x d y \leq \sum_{k=1}^{N} \int_{2 B_{k}} \int_{B\left(y, 2^{-m+3}\right)} 2^{m p}|x-y|^{p-2-s p} d x d y \\
\leq c \sum_{k=1}^{N}\left|B_{k}\right| 2^{m p} 2^{-m(p-s p)} \leq c 2^{\lambda m} 2^{-2 m} 2^{m p} 2^{-m(p-s p)} \leq c ;
\end{gathered}
$$

recall here that $s p=2-\lambda$. For $\ell=2,3, \ldots$, we have

$$
\begin{aligned}
& \sum_{k=1}^{N} \int_{(\ell+1) B_{k} \backslash \ell B_{k}} \int_{B_{k}} \frac{\left|u_{m}(x)-u_{m}(y)\right|^{p}}{|x-y|^{2+s p}} d x d y \\
& \quad \leq c \sum_{k=1}^{N}\left|(\ell+1) B_{k} \backslash \ell B_{k}\right| \cdot\left|B_{k}\right| \frac{1}{\left(\ell 2^{-m}\right)^{2+s p}} \\
& \quad \leq c 2^{\lambda m}\left(\ell 2^{-m}\right) 2^{-m}\left|B_{k}\right| 2^{m(2+s p)} \frac{1}{\ell^{2+s p}} \leq c 2^{m(\lambda-2-2+2+s p)} \ell^{-(1+s p)} \leq c \ell^{-(1+s p)} .
\end{aligned}
$$

Since $s p>0$, it follows that

$$
I_{1} \leq c \sum_{\ell=0}^{\infty} \sum_{k=1}^{N} \int_{(\ell+1) B_{k} \backslash \ell B_{k}} \int_{B_{k}} \frac{\left|u_{m}(x)-u_{m}(y)\right|^{p}}{|x-y|^{2+s p}} d x d y \leq c+\sum_{\ell=2}^{\infty} \ell^{-(1+s p)} \leq c .
$$

Let us then estimate the integral $I_{2}$. We write

$$
Q_{m}^{2}=\left(([-2,2] \times[0,2]) \backslash E_{m}\right) \cap G \quad \text { and } \quad Q_{m}^{3}=\left(([-3,3] \times[0,3]) \backslash E_{m}\right) \cap G .
$$

Then, with simple manipulations and the fact that $u_{m}=0$ outside of the set $[-2,2] \times$ $[0,2]$, we find

$$
\begin{aligned}
I_{2} & =\int_{G \backslash E_{m}} \int_{G \backslash E_{m}} \frac{\left|u_{m}(x)-u_{m}(y)\right|^{p}}{|x-y|^{2+s p}} d x d y \\
& \leq\left\{\int_{Q_{m}^{3}} \int_{Q_{m}^{2}}+2 \int_{Q_{m}^{2}} \int_{G \backslash\left(Q_{m}^{3} \cup E_{m}\right)}\right\} \frac{\left|u_{m}(x)-u_{m}(y)\right|^{p}}{|x-y|^{2+s p}} d x d y=: I_{21}+2 I_{22} .
\end{aligned}
$$

By the properties of snowflake curves, the set $Q_{m}^{3}$ is quasiconvex; that is, all pairs $x, y \in Q_{m}^{3}$ can be joined by a curve $\gamma_{x, y} \subset Q_{m}^{3}$ such that length $(\gamma) \leq c|x-y|$. Thus $\left|u_{m}(x)-u_{m}(y)\right| \leq c|x-y|$ for all $x, y \in Q_{m}^{3}$ by property (4) of the functions $u_{m}$, and so

$$
I_{21} \leq c \int_{Q_{m}^{3}} \int_{Q_{m}^{2}} \frac{|x-y|^{p}}{|x-y|^{2+s p}} d x d y \leq c \int_{Q_{m}^{3}} \int_{B(y, 10)}|x-y|^{p-2-s p} d x d y \leq c .
$$


Finally,

$$
\begin{aligned}
I_{22} & =\int_{Q_{m}^{2}} \int_{G \backslash\left(Q_{m}^{3} \cup E_{m}\right)} \frac{\left|u_{m}(x)-u_{m}(y)\right|^{p}}{|x-y|^{2+s p}} d x d y \\
& \leq \int_{Q_{m}^{2}} \int_{G \backslash B(y, 1)} \frac{1}{|x-y|^{2+s p}} d x d y \leq c,
\end{aligned}
$$

where we used also the fact that $[-3,3] \times[0,-1] \subset G^{c}$. By the above calculations we conclude that, indeed,

$$
\int_{G} \int_{G} \frac{\left|u_{m}(x)-u_{m}(y)\right|^{p}}{|x-y|^{2+s p}} d x d y \leq C
$$

where the constant $C>0$ is independent of $m$. Consequently, $G$ can not admit $(s, p)$-Hardy inequalities for $s p=2-\lambda$.

5.6. Local counterexample for $s p=1$. Let us now consider a domain $G \subset \mathbb{R}^{2}$ such that $G=G^{\text {core }} \backslash K$, where $G^{\text {core }}$ is a snowflake domain with $\operatorname{dim}_{\mathcal{H}}\left(\partial G^{\text {core }}\right)=$ $\lambda \in(1,2)$ (cf. Section 5.2) and $K$ is a fat Cantor set (cf. Section 5.3) placed inside $G^{\text {core }}$ in such a way that $\operatorname{dist}\left(K, \partial G^{\text {core }}\right) \geq c_{0}>0$. Then the boundary of $G$ is locally $(s, p)$-uniformly fat whenever $s p>2-\lambda$, and so in particular for $s p=1$, but we will show that $G$ can not admit $(s, p)$-Hardy inequalities when $s p=1$.

For simplicity, we may assume that $K=K\left(Q_{0}\right)$ with $Q_{0}=[0,1] \times[0,1]$, as constructed in Section 5.2, and that $\operatorname{dist}\left(K, \partial G^{\text {core }}\right) \geq 1$. For each $k \geq 0$, let $Q_{k}=Q_{0, k, m}$ be the $k$ th level cube in the construction of $K$ (with side length $\ell_{k}=\ell_{0, k}$ ) satisfying $(0,1) \in Q_{k}$. The idea is to place above each $Q_{k}$ a scaled copy of a suitable function $u_{m}, m=m(k)$, from Section 5.5 with $\lambda=1$. More precisely, the cube $\left[0, \ell_{k}\right] \times\left[1,1+\ell_{k} / 2\right]$ corresponds now to the cube $[-3,3] \times[0,3]$; the line segment from $(0,1)$ to $\left(0+\ell_{k}, 1\right)$ corresponds to $K_{1}$ of Section 5.5 ; and the functions $u_{m}$ are scaled accordingly. If $2^{-m} \geq \varepsilon_{k}$, where $\varepsilon_{k}=\varepsilon_{0, k}$ gives the relative width of the 'corridor' in the $(k+1)$ th level of the construction of $K$, then estimate (5.12), when applied to these scaled copies of $u_{m}$ in $\left[0, \ell_{k}\right] \times\left[1,1+\ell_{k} / 2\right]$, yields

$$
\int_{G} \frac{\left|u_{m}(x)\right|^{p}}{\operatorname{dist}(x, \partial G)^{s p}} d x \geq c \ell_{k}^{2-s p} m .
$$

Notice, however, that for a fixed $k$ this estimate is not true when $m \rightarrow \infty$, since then also the small corridors in $Q_{k} \backslash K$ influence the factor $\operatorname{dist}(x, \partial G)$ in the integral.

The terms on the right-hand side of the $(s, p)$-Hardy inequality are scaled with the factor $\ell_{k}^{2-s p}$, too. Estimates for the integrals corresponding to $I_{1}$ and $I_{21}$ from Section 5.5 are valid as such, and thus these terms are bounded by $c \ell_{k}^{2-s p}$. However, in estimate (5.13) for the integral $I_{22}$ we used the fact that $[-3,3] \times[0,-1] \subset G^{c}$, but the corresponding claim is clearly not true in the present setting; in fact, $Q_{k} \cap G$ is even a dense set. To overcome this, we need to be a bit more careful when estimating this last integral.

We choose $\varepsilon_{k}=2^{-k(2+s p)} / 4$, and thus $\sum_{k^{\prime}=k}^{\infty} 2 \varepsilon_{k^{\prime}} \simeq \varepsilon_{k}$ for all $k \geq 0$. By Lemma 5.5, we know that then

$$
\left|Q_{k} \cap G\right| \leq\left|Q_{k}\right| \sum_{k^{\prime}=k}^{\infty} 2 \varepsilon_{k^{\prime}} \simeq \ell_{k}^{2} \varepsilon_{k} \simeq \ell_{k}^{2} 2^{-k(2+s p)} .
$$


Let $\hat{Q}_{m}^{2}, \hat{Q}_{m}^{3} \subset\left[0, \ell_{k}\right] \times\left[1,1+\ell_{k} / 2\right]$ denote the obvious analogs of $Q_{m}^{2}$ and $Q_{m}^{3}$. Then we can estimate the integral corresponding to $I_{22}$ as

$$
\begin{aligned}
& \int_{\hat{Q}_{m}^{2}} \int_{G \backslash \hat{Q}_{m}^{3}} \frac{\left|u_{m}(x)-u_{m}(y)\right|^{p}}{|x-y|^{2+s p}} d x d y \\
& \quad \leq \int_{\hat{Q}_{m}^{2}} \int_{G \backslash B\left(y, \ell_{k} / 6\right)} \frac{1}{|x-y|^{2+s p}} d x d y+\int_{\hat{Q}_{m}^{2}} \int_{Q_{k} \cap G} \frac{1}{|x-y|^{2+s p}} d x d y \\
& \quad \leq c \ell_{k}^{2-s p}+\left|Q_{k}\right|\left|Q_{k} \cap G\right| 2^{m(2+s p)} \ell_{k}^{-(2+s p)} \\
& \quad \leq c \ell_{k}^{2-s p}+\ell_{k}^{2-s p} 2^{-k(2+s p)} 2^{m(2+s p)}
\end{aligned}
$$

where we used (5.15) and the fact that $|x-y| \geq \ell_{k} 2^{-m}$ when $y \in \hat{Q}_{m}^{2}$ and $x \in$ $Q_{k} \cap G$. Thus, for $m=m(k)=k$, the integral above is bounded by $\ell_{k}^{2-s p}$, as desired. Moreover, with this choice we have $2^{-m(k)}=2^{-k} \geq 2^{-k(2+s p)} \geq \varepsilon_{k}$, which was the only requirement for estimate (5.14) to hold. In conclusion, the $(s, p)$-Hardy inequality can not be valid for functions $u_{m(k)}, m(k)=k$, with a uniform constant, and hence the domain $G$ can not admit $(s, p)$-Hardy inequalities when $s p=1$.

5.7. Local snowflaked counterexamples for $0<s p<1$. Let us briefly indicate how to obtain similar counterexamples when $0<s p<1$. The value $s p=1$ above corresponds to the fact that the fat Cantor set is, loosely speaking, seen as onedimensional when observed from a suitable distance from within the domain; here $1=2-s p$. Similarly, if we had a locally $(s, p)$-uniformly fat part of the boundary that is seen as $\lambda$-dimensional, with $\lambda=2-s p$, then $(s, p)$-Hardy inequalities would fail. Such a situation can be obtained, for instance, by placing a snowflake type set $F_{\lambda}$, indicated in Figure 3, inside a suitable $G^{\text {core }}$-domain. Notice, however, that the set in Figure 3 is based on a very simple approximation of the actual snowflake curve $K_{\lambda}$.

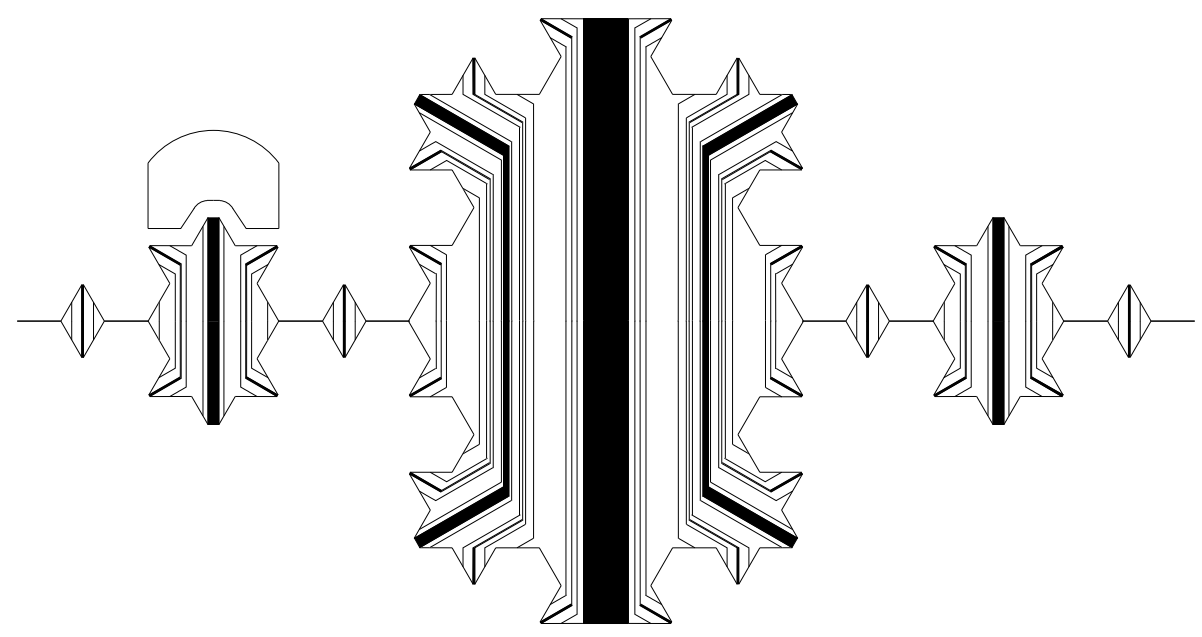

FigurE 3. Snowflake set $F_{\lambda}$ with tunnels. The support of the test function $u_{2}$ is drawn above the snowflake rhombus $Q_{2}$.

More precisely, to obtain such a set $F_{\lambda}$, we first consider the set bounded by a snowflake curve $K_{\lambda}$ of dimension $\lambda \in(1,2)$ and its reflection on the $x_{1}$-axis, and then remove 'tunnels' (the black parts in Figure 3) of width $\varepsilon_{k}$ : one large tunnel of width $\varepsilon_{1}$, four tunnels of width $\varepsilon_{2}$, etc. It is clear that if we choose $\varepsilon_{k}>0$ to be small 
enough, then the resulting set $F_{\lambda}$ is still 2-regular, and thus locally $(s, p)$-uniformly fat for any $1<p<\infty$ and $0<s<1$. If the boundary of the surrounding $G^{\text {core }}{ }_{-}$ domain has dimension greater than $\lambda$, it follows that the boundary of $G=G^{\text {core }} \backslash F_{\lambda}$ is locally $(s, p)$-uniformly fat for $s p=2-\lambda$.

Now, let $Q_{1} \subset \partial G$ denote the large 'snowflake rhombus' in the middle of $K_{\lambda}$. Then choose $Q_{2}$ to be the second largest rhombus on the left-hand side of $Q_{1}$; and $Q_{3}$ to be the third largest rhombus on the left-hand side of $Q_{2}$; and so on. Just like in the counterexample of Section 5.6 with the fat Cantor set, we place scaled copies of the functions $u_{m}$ from Section 5.5 (constructed with respect to the corresponding $K_{\lambda}$ ) above the sets $Q_{k}$; cf. Figure 3. With computations analogous to those in Section 5.6 (we leave the details to the reader), it follows that, for $s p=2-\lambda, G$ can not admit $(s, p)$-Hardy inequalities, and yet $G$ is a domain having a locally $(s, p)$-uniformly fat boundary. This finishes the proof of the second claim of Theorem 5.1.

\section{Applications}

We conclude the paper with extension and removability problems related to fractional Hardy inequalities and fractional Sobolev spaces. For $0<s<1$ and $1<p<\infty$, the fractional Sobolev space $W^{s, p}(G)$ is defined as the space of functions $u \in L^{p}(G)$ with $\|u\|_{W^{s, p}(G)}:=\|u\|_{L^{p}(G)}+|u|_{W^{s, p}(G)}<\infty$, where

$$
|u|_{W^{s, p}(G)}:=\left(\int_{G} \int_{G} \frac{|u(x)-u(y)|^{p}}{|x-y|^{n+s p}} d y d x\right)^{1 / p} .
$$

See [4] for a nice introduction and basic properties of the spaces $W^{s, p}(G)$.

6.1. Zero Extension. We recall here a straightforward but useful connection between boundedness of certain extension operators and Hardy inequalities [14]. For an open set $G \subset \mathbb{R}^{n}$, we define the 'zero extension operator' $E_{G}$ as

$$
E_{G}: L^{p}(G) \rightarrow L^{p}\left(\mathbb{R}^{n}\right), \quad E_{G} u(x)= \begin{cases}u(x), & x \in G ; \\ 0, & x \in \mathbb{R}^{n} \backslash G .\end{cases}
$$

We say that the operator $E_{G}$ is bounded, if there is a constant $c_{1}>0$ such that

$$
\left|E_{G} u\right|_{W^{s, p}\left(\mathbb{R}^{n}\right)}^{p} \leq c_{1}|u|_{W^{s, p}(G)}^{p}
$$

for every $u \in C_{0}^{\infty}(G)$.

Lemma 6.3. Let $0<s<1$ and $1<p<\infty$, and suppose that $G \subset \mathbb{R}^{n}$ is an open set. Then the zero extension $E_{G} u$ of any $u \in W^{s, p}(G)$ satisfies the inequality

$$
\left|E_{G} u\right|_{W^{s, p}\left(\mathbb{R}^{n}\right)}^{p} \leq|u|_{W^{s, p}(G)}^{p}+C \int_{G} \frac{|u(x)|^{p}}{\operatorname{dist}(x, \partial G)^{s p}} d x,
$$

where the constant $C$ depends on $n, s$, and $p$.

Proof. Since

$$
\begin{aligned}
\left|E_{G} u\right|_{W^{s, p}\left(\mathbb{R}^{n}\right)}^{p} & =\int_{\mathbb{R}^{n}} \int_{\mathbb{R}^{n}} \frac{\left|E_{G} u(x)-E_{G} u(y)\right|^{p}}{|x-y|^{n+s p}} d y d x \\
& \leq|u|_{W^{s, p}(G)}^{p}+2 \int_{G}|u(x)|^{p} \int_{G^{c}} \frac{1}{|x-y|^{n+s p}} d y d x
\end{aligned}
$$


it remains to apply the following estimates, which are valid for $x \in G$ :

$$
\begin{aligned}
\int_{G^{c}} \frac{1}{|x-y|^{n+s p}} d y & \leq \int_{\mathbb{R}^{n} \backslash B(x, \operatorname{dist}(x, \partial G))} \frac{1}{|x-y|^{n+s p}} d y \\
& \leq C \int_{\operatorname{dist}(x, \partial G)}^{\infty} r^{-1-s p} d r \leq C \operatorname{dist}(x, \partial G)^{-s p} .
\end{aligned}
$$

This completes the proof of the lemma.

We also need the following auxiliary Hardy-type inequality, an adaptation of results in [9] and [13, Theorem 3.1].

Theorem 6.5. Let $1<p<\infty$ and $0<s<1$ be such that $s p<n$. Let $G \subset \mathbb{R}^{n}$ be an open set, $n \geq 2$, whose complement is $(s, p)$-uniformly fat. Then, for every $u \in C_{0}^{\infty}(G)$,

$$
\int_{G} \frac{|u(x)|^{p}}{\operatorname{dist}(x, \partial G)^{s p}} d x \leq c_{0} \int_{\mathbb{R}^{n}} \int_{\mathbb{R}^{n}} \frac{\left|E_{G} u(x)-E_{G} u(y)\right|^{p}}{|x-y|^{n+s p}} d y d x,
$$

where the constant $c_{0}$ depends on $s, n, p$, and the fatness constant $\sigma$.

With the help of the above results, we establish a connection between extensions and Hardy inequalities:

Theorem 6.6. Let $1<p<\infty$ and $0<s<1$ be such that sp $<n$. Let $G \subset \mathbb{R}^{n}$, $n \geq 2$, be an open set. Then the following two statements hold:

(1) If $G^{c}$ is $(s, p)$-uniformly fat and $E_{G}$ is bounded, then $G$ admits an $(s, p)$-Hardy inequality.

(2) Conversely, suppose that $G$ admits an $(s, p)$-Hardy inequality. Then $E_{G}$ is bounded.

Proof. First suppose that the assumptions in (1) hold. By Theorem 6.5 we have for every $u \in C_{0}^{\infty}(G)$ that

$$
\int_{G} \frac{|u(x)|^{p}}{\operatorname{dist}(x, \partial G)^{s p}} d x \leq c_{0}\left|E_{G} u\right|_{W^{s, p}\left(\mathbb{R}^{n}\right)}^{p} \leq c_{0} c_{1}|u|_{W^{s, p}(G)}^{p} .
$$

Hence, the $(s, p)$-Hardy inequality holds with constant $c_{2}=c_{0} c_{1}$.

Suppose then that the assumption in (2) holds; in other words, there is $c_{2}>0$ such that, for every $u \in C_{0}^{\infty}(G)$,

$$
\int_{G} \frac{|u(x)|^{p}}{\operatorname{dist}(x, \partial G)^{s p}} d x \leq c_{2}|u|_{W^{s, p}(G)}^{p} .
$$

By Lemma 6.3 we then have

$$
\left|E_{G} u\right|_{W^{s, p}\left(\mathbb{R}^{n}\right)}^{p} \leq|u|_{W^{s, p}(G)}^{p}+C \int_{G} \frac{|u(x)|^{p}}{\operatorname{dist}(x, \partial G)^{s p}} d x \leq\left(1+C c_{2}\right)|u|_{W^{s, p}(G)}^{p}
$$

for every $u \in C_{0}^{\infty}(G)$, and thus $E_{G}$ is bounded.

The following corollary is an immediate consequence of Corollary 1.4 and Theorem 6.6.

Corollary 6.7. Let $1<p<\infty$ and $0<s<1$ be such that $s p<n$. Suppose that $G \subset \mathbb{R}^{n}$ is a (bounded) uniform domain whose boundary is (locally) $(s, p)$-uniformly fat. Then $E_{G}$ is bounded. 
This corollary applies for instance to the usual Koch snowflake domain $G$ with $2-s p<\log 4 / \log 3$.

6.2. Removability. Finally, let us record the following removability result for fractional Hardy inequalities.

Theorem 6.8. Let $1<p<\infty$ and $0<s<1$ be such that $s p<n$. Assume that $G^{\prime} \subset G \subset \mathbb{R}^{n}$ are open sets such that $\left(G^{\prime}\right)^{c}$ is $(s, p)$-uniformly fat, $G$ admits an $(s, p)$-Hardy inequality, and $\left|G \backslash G^{\prime}\right|=0$. Then $G^{\prime}$ admits an $(s, p)$-Hardy inequality.

Proof. By Theorem 6.6(2), there is a constant $c_{1}>0$ such that, for every $g \in C_{0}^{\infty}(G)$,

$$
\left|E_{G} g\right|_{W^{s, p}\left(\mathbb{R}^{n}\right)}^{p} \leq c_{1}|g|_{W^{s, p}(G)}^{p} .
$$

Let $u \in C_{0}^{\infty}\left(G^{\prime}\right)$, and define $g:=\left.\left(E_{G^{\prime}} u\right)\right|_{G} \in C_{0}^{\infty}(G)$. An application of the previous inequality (6.9) to this function yields

$$
\begin{aligned}
\left|E_{G^{\prime}} u\right|_{W^{s, p}\left(\mathbb{R}^{n}\right)}^{p} & =\left|E_{G} g\right|_{W^{s, p}\left(\mathbb{R}^{n}\right)}^{p} \leq c_{1}|g|_{W^{s, p}(G)}^{p}=\left.c_{1}\left|\left(E_{G^{\prime}} u\right)\right|_{G}\right|_{W^{s, p}(G)} ^{p} \\
& =c_{1} \int_{G} \int_{G} \frac{\left|E_{G^{\prime}} u(x)-E_{G^{\prime}} u(y)\right|^{p}}{|x-y|^{n+s p}} \\
& =c_{1} \int_{G^{\prime}} \int_{G^{\prime}} \frac{|u(x)-u(y)|^{p}}{|x-y|^{n+s p}}=c_{1}|u|_{W^{s, p}\left(G^{\prime}\right)}^{p} .
\end{aligned}
$$

In the penultimate step, we used the assumption that $\left|G \backslash G^{\prime}\right|=0$. As a conclusion, we have shown that $\left|E_{G^{\prime}} u\right|_{W^{s, p}\left(\mathbb{R}^{n}\right)}^{p} \leq c_{1}|u|_{W^{s, p}\left(G^{\prime}\right)}^{p}$ for every $u \in C_{0}^{\infty}\left(G^{\prime}\right)$. By Theorem 6.6, we find that $G^{\prime}$ admits an $(s, p)$-Hardy inequality.

Remark 6.10. Let us indicate a possible application of Theorem 6.8. By Corollary 1.4 , we may begin with a uniform domain $G \subset \mathbb{R}^{n}$ whose boundary is $(s, p)$-uniformly fat with $1<p<\infty$ and $0<s p<n$. Let $K \subset G$ be a closed $(s, p)$-uniformly fat set with zero measure. Then it follows from Theorem 6.8 that the open set $G^{\prime}:=G \backslash K$ admits an $(s, p)$-Hardy inequality.

Acknowledgements. The research is supported by the Academy of Finland, grants no. 135561 (L.I. and H.T.) and 252108 (J.L.).

\section{REFERENCES}

[1] D. R. Adams and L. I. Hedberg, Function spaces and potential theory, Grundlehren Math. Wiss. 314, Springer-Verlag, Berlin, 1996.

[2] K. Bogdan and B. Dyda, The best constant in a fractional order Hardy inequality, Math. Nachr. 284 (2011), 629-638.

[3] L. Carleson, Selected problems on exceptional sets, Van Nostrand Mathematical Studies, No. 13 D. Van Nostrand Co., Inc., Princeton, N.J.-Toronto, Ont.London, 1967.

[4] E. Di Nezza, G. Palatucci, and E. Valdinoci, Hitchhiker's guide to the fractional Sobolev spaces, Bull. Sci. Math. 136 (2012), 521-573.

[5] B. Dyda, A fractional order Hardy inequality, Illinois J. Math. 48 (2004), 575588. 
[6] B. Dyda, Fractional Hardy inequality with a remainder term, Colloq. Math. 122 (2011), 59-67.

[7] B. Dyda and R. L. Frank, Fractional Hardy-Sobolev-Maz'ya inequality for domains, Studia Math. 208 (2012), 151-166.

[8] B. Dyda and A. V. Vähäkangas, A framework for fractional Hardy inequalities, Ann. Acad. Sci. Fenn. Math. 39 (2014), 675-689.

[9] D. E. Edmunds, R. Hurri-Syrjänen and A. V. Vähäkangas, Fractional Hardytype inequalities in domains with uniformly fat complement, Proc. Amer. Math. Soc. 142 (2014), 897-907.

[10] S. Filippas, L. Moschini, and A. Tertikas, Sharp trace Hardy-Sobolev-Maz'ya inequalities and the fractional Laplacian, Arch. Ration. Mech. Anal. 208 (2013), 109-161.

[11] R. L. Frank and R. Seiringer, Sharp Fractional Hardy Inequalities in HalfSpaces, Around the research of Vladimir Maz'ya. I, 161-167, Int. Math. Ser. (N. Y.), 11, Springer, New York, 2010.

[12] R. Hurri-Syrjänen and A. V. Vähäkangas, On fractional Poincaré inequalities, J. Anal. Math. 120 (2013), 85-104.

[13] L. Ihnatsyeva and A. V. Vähäkangas, Hardy inequalities in Triebel-Lizorkin spaces, Indiana Univ. Math. J. 62 (2013), 1785-1807.

[14] L. Ihnatsyeva and A. V. Vähäkangas, Hardy inequalities in TriebelLizorkin spaces II. Aikawa dimension, Ann. Mat. Pura Appl. (4) (2013), DOI:10.1007/s10231-013-0385-z.

[15] R. Korte, J. Lehrbäck and H. Tuominen, The equivalence between pointwise Hardy inequalities and uniform fatness, Math. Ann. 351 (2011), 711-731.

[16] P. Koskela, The degree of regularity of a quasiconformal mapping, Proc. Amer. Math. Soc. 122 (1994), 769-772.

[17] P. Koskela and J. Lehrbäck, Weighted pointwise Hardy inequalities, J. London Math. Soc. 79 (2009), 757-779.

[18] A. Kufner and L. E. Persson, Weighted inequalities of Hardy type, World Scientific Publishing Co., New Jersey, 2003.

[19] J. Lehrbäck, Pointwise Hardy inequalities and uniformly fat sets, Proc. Amer. Math. Soc. 136 (2008), 2193-2200.

[20] J. Lehrbäck, Weighted Hardy inequalities and the size of the boundary, Manuscripta Math. 127 (2008), 249-273.

[21] J. Lehrbäck, Weighted Hardy inequalities beyond Lipschitz domains, Proc. Amer. Math. Soc. 142 (2014), 1705-1715.

[22] J. L. Lewis, Uniformly fat sets, Trans. Amer. Math. Soc. 308 (1988), 177-196.

[23] M. Loss and C. A. Sloane, Hardy inequalities for fractional integrals on general domains, J. Funct. Anal. 259 (2010), 1369-1379. 
[24] P. Mattila, Geometry of sets and measures in Euclidean spaces. Fractals and rectifiability, Cambridge Studies in Advanced Mathematics, 44. Cambridge University Press, Cambridge, 1995.

[25] J. Nečas, Sur une méthode pour résoudre les équations aux dérivées partielles du type elliptique, voisine de la variationnelle, Ann. Scuola Norm. Sup. Pisa (3) 16 (1962), 305-326.

[26] C. A. Sloane, A fractional Hardy-Sobolev-Maz'ya inequality on the upper halfspace, Proc. Amer. Math. Soc. 139 (2011), 4003-4016.

[27] E. M. Stein, Singular integrals and differentiability properties of functions, Princeton Univ. Press, Princeton, New Jersey, 1970.

[28] A. Wannebo, Hardy inequalities, Proc. Amer. Math. Soc. 109 (1990), 85-95.

(L.I.) Aalto University, Department of Mathematics and Systems Analysis, P.O. Box 11100, FI-00076 Aalto, Finland, and University of Jyvaskyla, Department of Mathematics and Statistics, P.O. Box 35 (MaD), FI-40014 University of Jyvaskyla, FINLAND

E-mail address: lizaveta.ihnatsyeva@aalto.fi

(J.L.) University of Jyvaskyla, Department of Mathematics and Statistics, P.O. Box 35 (MaD), FI-40014 University of Jyvaskyla, Finland

E-mail address: juha.lehrback@jyu.fi

(H.T.) University of Jyvaskyla, Department of Mathematics and Statistics, P.O. Box 35 (MaD), FI-40014 University of Jyvaskyla, Finland

E-mail address: heli.m.tuominen@jyu.fi

(A.V.V.) University of Helsinki, Department of Mathematics and Statistics, P.O. Box 68, FI-00014 University of Helsinki, Finland, and University of Jyvaskyla, Department of Mathematics and Statistics, P.O. Box 35 (MaD), FI-40014 University of Jyvaskyla, Finland

E-mail address: antti.vahakangas@helsinki.fi 